Acta Crystallographica Section E

Structure Reports

Online

ISSN 1600-5368
S. E. Duff, ${ }^{\text {a }}$ P. B. Hitchcock, ${ }^{\text {b }}$

S. C. Davies, ${ }^{a}$ J. E. Barclay ${ }^{a}$ and

D. J. Evans ${ }^{\mathrm{a} *}$

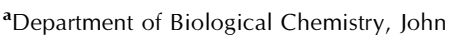
Innes Centre, Norwich Research Park, Colney, Norwich NR4 7UH, England, and ${ }^{\mathbf{b}}$ Department of Chemistry, University of Sussex, Falmer, Brighton BN1 9QJ, England

Correspondence e-mail:

dave.evans@bbsrc.ac.uk

\section{Key indicators}

Single-crystal X-ray study

$T=173 \mathrm{~K}$

Mean $\sigma(\mathrm{C}-\mathrm{C})=0.009 \AA$

$\mathrm{H}$-atom completeness $93 \%$

$R$ factor $=0.052$

$w R$ factor $=0.107$

Data-to-parameter ratio $=11.6$

For details of how these key indicators were automatically derived from the article, see http://journals.iucr.org/e.

\title{
1,3-Bis(diphenylphosphino)propane-2 $\kappa^{2} P, P^{\prime}-$ dicarbonyl-1 $\kappa^{2} C$-chloro-2 $\kappa C l-\left\{\mu\right.$-2, $2^{\prime}, 2^{\prime \prime}$-nitrilo- triethanethiolato(3-)-1 $\left.\kappa^{4} N, S, S^{\prime}, S^{\prime \prime}: 2 \kappa^{2} S, S^{\prime}\right\}$ - iron(II)nickel(II) acetonitrile solvate
}

The structure of the neutral title complex, [[Fe$\left.\left.\left[\left(\mathrm{SCH}_{2} \mathrm{CH}_{2}\right)_{3} \mathrm{~N}\right](\mathrm{CO})_{2}-\mathrm{S}, \mathrm{S}^{\prime}\right\} \mathrm{NiCl}\left\{\left[\mathrm{P}\left(\mathrm{C}_{6} \mathrm{H}_{5}\right)_{2}\right]_{2}\left(\mathrm{CH}_{2}\right)_{3}\right\}\right] \cdot \mathrm{CH}_{3} \mathrm{CN}$ or $\left[\mathrm{FeNi}\left(\mathrm{C}_{6} \mathrm{H}_{12} \mathrm{NS}_{3}\right) \mathrm{Cl}\left(\mathrm{C}_{27} \mathrm{H}_{26} \mathrm{P}_{2}\right)(\mathrm{CO})_{2}\right] \cdot \mathrm{C}_{2} \mathrm{H}_{3} \mathrm{~N}$, is described. There are two independent complex molecules and two solvent molecules in the asymmetric unit. The $\mathrm{Fe}$ atoms are octahedrally coordinated; the three $\mathrm{S}$ atoms and a $\mathrm{C}$ atom of one of the two $\mathrm{CO}$ ligands form the equatorial plane, with the $\mathrm{N}$ atom of the $\left(\mathrm{SCH}_{2} \mathrm{CH}_{2}\right)_{3} \mathrm{~N}$ ligand and the second carbonyl $\mathrm{C}$ atom lying in the axial positions. The $\mathrm{Ni}$ atoms are square pyramidally coordinated, with the two bridging $\mathrm{S}$ atoms and the $\mathrm{P}$ atoms of the 1,3-bis(diphenylphosphino)propane ligand forming the basal plane and the $\mathrm{Cl}$ atom lying in the apical position.

\section{Comment}

The title compound, (I), was prepared as a further example of a synthetic structural analogue of the dimetallic active site of the enzyme nickel-iron hydrogenase (Evans \& Pickett, 2003; Davies et al., 1999; Smith et al., 2002, 2003). Compound (I) is closely related to $\left[\left\{\mathrm{Fe}\left[\left(\mathrm{SCH}_{2} \mathrm{CH}_{2}\right)_{3} \mathrm{~N}\right](\mathrm{CO})_{2}-\mathrm{S}, \mathrm{S}^{\prime}\right\} \mathrm{NiCl}-\right.$ $\left.\left\{\left[\mathrm{P}\left(\mathrm{C}_{6} \mathrm{H}_{5}\right)_{2}\right]_{2}\left(\mathrm{CH}_{2}\right)_{2}\right\}\right]$, (II) (Davies et al., 1999; Smith et al., 2002), in which the chelating diphosphane is 1,2-bis(diphenylphosphino)ethane (dppe), whereas in (I) it is 1,3-bis(diphenylphosphino)propane (dppp).

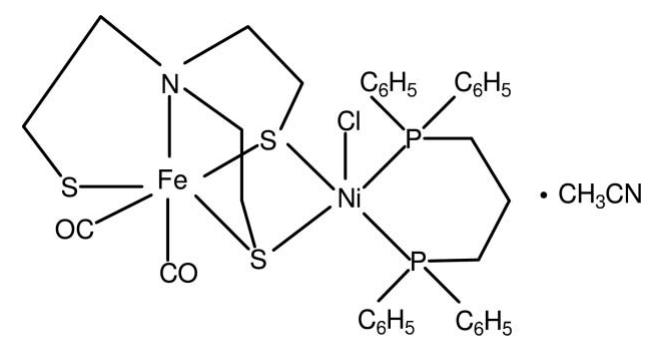

(I)

The asymmetric unit consists of two complex molecules and two solvent molecules. The $\mathrm{S}_{3} \mathrm{C}$ equatorial planes of the octahedrally coordinated Fe atoms are slightly distorted, with deviations from the mean planes lying in the range -0.069 (3) to 0.074 (3) $\AA$ in molecule 1 (the negative sign indicates the opposite side of the mean plane) and -0.060 (3) to 0.063 (3) $\AA$ in molecule 2. The $\mathrm{Fe}$ atoms are displaced from these mean equatorial planes by 0.067 (2) and 0.1400 (12) A, respectively, towards the axial $\mathrm{CO}$ ligand. The $\mathrm{S}_{2} \mathrm{P}_{2}$ basal planes of the square pyramidally coordinated $\mathrm{Ni}$ atoms are also slightly distorted, with deviations from the mean planes lying in the range -0.054 (3) to 0.053 (3) $\AA$ in molecule 1 and -0.035 (3) to 0.035 (3) $\AA$ in molecule 2 . The $\mathrm{Ni}$ atoms are displaced 0.3227 (13) and 0.3175 (14) $\AA$, respectively, from these mean
Received 20 May 2005 Accepted 7 June 2005

Online 17 June 2005 


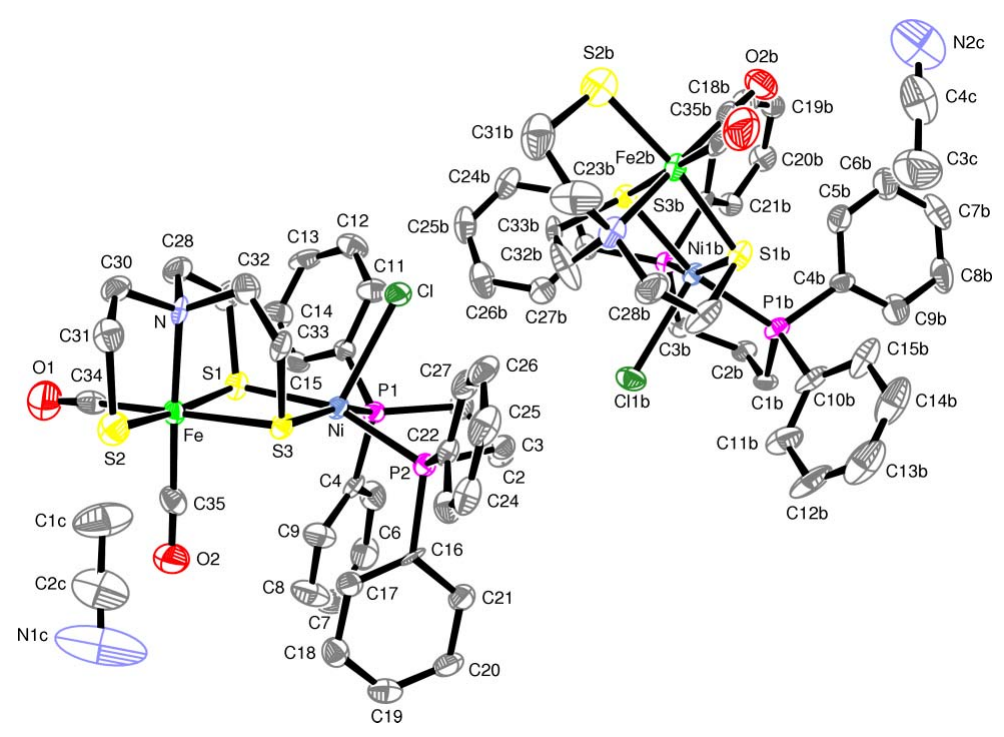

Figure 1

The asymmetric unit of (I), showing displacement ellipsoids at the $50 \%$ probability level. $\mathrm{H}$ atoms have been omitted.

planes, towards the $\mathrm{Cl}$ atoms. The $\mathrm{S}_{3} \mathrm{C}$ and $\mathrm{S}_{2} \mathrm{P}_{2}$ mean planes are not coplanar, with an angle between their normals of $16.31(6)^{\circ}$; in (II), this angle is $19.38(7)^{\circ}$.

Bond dimensions about the $\mathrm{Fe}$ and $\mathrm{Ni}$ atoms are not unusual and are comparable to those in (II) [see Table 1 for dimensions in (I)]. The $\mathrm{Fe}-\mathrm{C}$ bond to the equatorial $\mathrm{CO}$ ligand is slightly longer than those to the axial CO ligand in both molecules of (I) and in complex (II); in the second molecule of (I) it is longer than in the first and in (II) as a result of unresolved disorder in the $\mathrm{O}$ atom $\left[\mathrm{Fe}-\mathrm{C}_{\mathrm{eq}}=\right.$ 1.783 (12) $\AA$ and $\mathrm{Fe}-\mathrm{C}_{\text {axial }}=1.741$ (10) in (II)].

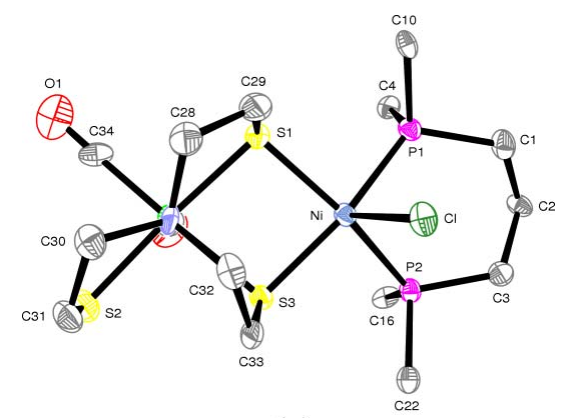

(a)

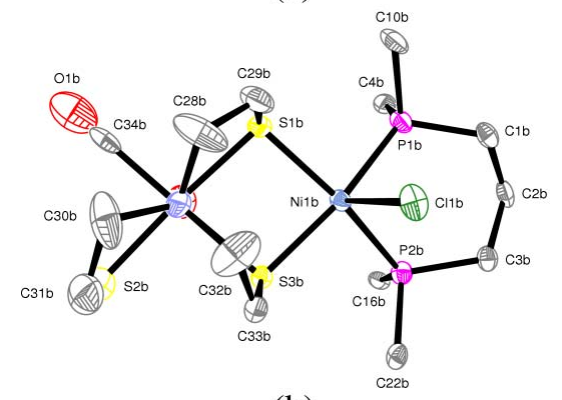

(b)

Figure 2

View along the $\mathrm{N}-\mathrm{Fe}-\mathrm{C}-\mathrm{O}$ axis of $(a)$ molecule 1 and $(b)$ molecule 2, showing the deviation from the pseudo-threefold rotation of the $\left(\mathrm{SCH}_{2} \mathrm{CH}_{2}\right)_{3} \mathrm{~N}$ ligand. $\mathrm{H}$ atoms have been omitted.

The unusual torsion angles in the $\left(\mathrm{SCH}_{2} \mathrm{CH}_{2}\right)_{3} \mathrm{~N}$ ligand in (I) are also found in (II); the usual pseudo-threefold rotation about the $\mathrm{Fe}-\mathrm{N}$ bond is removed by the non-bridging $\mathrm{SCH}_{2} \mathrm{CH}_{2}$ 'arm' in both structures (see Fig. 2). Other bond dimensions in the ligands are as expected.

The molecules are arranged within the crystal structure with the complex molecules forming chains parallel to the crystallographic $a$ axis; the solvent molecules occupy the centres of the channels enclosed by four such chains (see Fig. 3).

\section{Experimental}

Under an atmosphere of carbon monoxide, a mixture of $\left[\mathrm{NiCl}_{2}(\mathrm{dppp})\right](0.21 \mathrm{~g}, 0.39 \mathrm{mmol})$ and $\left(\mathrm{NEt}_{4}\right)\left[\mathrm{Fe}\left\{\left(\mathrm{SCH}_{2} \mathrm{CH}_{2}\right)_{3} \mathrm{~N}\right\}-\right.$ (CO)] $(0.16 \mathrm{~g}, 0.39 \mathrm{mmol})$ in $\mathrm{MeCN}(100 \mathrm{ml})$ was refluxed with stirring for $2.5 \mathrm{~h}$. After cooling overnight, crystals were collected by filtration and dried $(0.31 \mathrm{~g}, 97 \%)$. Analysis expected for $\mathrm{C}_{37} \mathrm{H}_{41} \mathrm{ClFeN}_{2} \mathrm{NiO}_{2} \mathrm{P}_{2} \mathrm{~S}_{3}$ : C 52.1, H 4.8, N 3.3\%; found C 51.7, H 4.9, N $3.2 \%$. v(CO), KBr: 1944 and $2006 \mathrm{~cm}^{-1}$; Mössbauer (solid, $80 \mathrm{~K}$, relative to iron foil at $298 \mathrm{~K}$ ) isomer shift $0.07 \mathrm{~mm} \mathrm{~s}^{-1}$, quadrupole splitting $0.56 \mathrm{~mm} \mathrm{~s}^{-1}$.

\section{Crystal data}

\section{$\left[\mathrm{FeNi}\left(\mathrm{C}_{6} \mathrm{H}_{12} \mathrm{NS}_{3}\right) \mathrm{Cl}\left(\mathrm{C}_{27} \mathrm{H}_{26} \mathrm{P}_{2}\right)-\right.$ $\left.(\mathrm{CO})_{2}\right] \cdot \mathrm{C}_{2} \mathrm{H}_{3} \mathrm{~N}$ \\ $M_{r}=853.85$ \\ Orthorhombic, $\mathrm{Pca}_{1}$

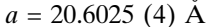 \\ $b=12.4769(2) \AA$ \\ $c=29.7090(6) \AA$ \\ $V=7636.9$ (2) $\AA^{3}$ \\ $Z=8$}

Data collection

Nonius KappaCCD diffractometer $\varphi$ and $\omega$ scans

Absorption correction: multi-scan (Blessing, 1995)

$T_{\min }=0.823, T_{\max }=0.979$

24708 measured reflections

10246 independent reflections

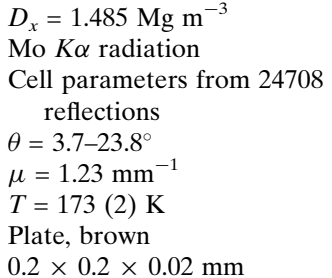

8837 reflections with $I>2 \sigma(I)$

$R_{\text {int }}=0.052$

$\theta_{\text {max }}=23.7^{\circ}$

$h=-22 \rightarrow 22$

$k=-14 \rightarrow 13$

$l=-27 \rightarrow 33$ 
Refinement

Refinement on $F^{2}$ $R\left[F^{2}>2 \sigma\left(F^{2}\right)\right]=0.052$

$w R\left(F^{2}\right)=0.107$

$S=1.07$

10246 reflections

884 parameters

$\mathrm{H}$-atom parameters constrained

\author{
$w=1 /\left[\sigma^{2}\left(F_{\mathrm{o}}^{2}\right)\right.$ \\ $+30.7123 P]$ \\ where $P=\left(F_{\mathrm{o}}{ }^{2}+2 F_{\mathrm{c}}{ }^{2}\right) / 3$ \\ $(\Delta / \sigma)_{\max }=0.006$ \\ $\Delta \rho_{\max }=0.88 \mathrm{e} \AA^{-3}$ \\ $\Delta \rho_{\min }=-0.63 \mathrm{e}^{-3}$ \\ Absolute structure: Flack (1983), \\ 4317 Friedel pairs \\ Flack parameter: $0.604(14)$
}

Table 1

Selected geometric parameters $\left(\AA{ }^{\circ}{ }^{\circ}\right)$.

\begin{tabular}{|c|c|c|c|}
\hline $\mathrm{Ni}-\mathrm{P} 1$ & $2.2012(14)$ & $\mathrm{Ni} 1 b-\mathrm{P} 1 b$ & $2.2018(15)$ \\
\hline $\mathrm{Ni}-\mathrm{P} 2$ & $2.2060(15)$ & $\mathrm{Ni} 1 b-\mathrm{P} 2 b$ & $2.2046(15)$ \\
\hline $\mathrm{Ni}-\mathrm{S} 3$ & 2.2517 (13) & $\mathrm{Ni} 1 b-\mathrm{S} 1 b$ & $2.2495(16)$ \\
\hline $\mathrm{Ni}-\mathrm{S} 1$ & $2.2574(15)$ & $\mathrm{Ni} 1 b-\mathrm{S} 3 b$ & $2.2536(14)$ \\
\hline $\mathrm{Ni}-\mathrm{Cl}$ & $2.5787(14)$ & $\mathrm{Ni} 1 b-\mathrm{Cl} 1 b$ & $2.5971(16)$ \\
\hline $\mathrm{Fe}-\mathrm{C} 35$ & $1.759(6)$ & $\mathrm{Fe} 2 b-\mathrm{C} 35 b$ & $1.760(6)$ \\
\hline $\mathrm{Fe}-\mathrm{C} 34$ & $1.829(6)$ & $\mathrm{Fe} 2 b-\mathrm{C} 34 b$ & $1.912(7)$ \\
\hline $\mathrm{Fe}-\mathrm{N}$ & $2.048(4)$ & $\mathrm{Fe} 2 b-\mathrm{N} 1 b$ & $2.065(5)$ \\
\hline $\mathrm{Fe}-\mathrm{S} 1$ & $2.2865(15)$ & $\mathrm{Fe} 2 b-\mathrm{S} 2 b$ & 2.2839 (19) \\
\hline $\mathrm{Fe}-\mathrm{S} 2$ & $2.2978(16)$ & $\mathrm{Fe} 2 b-\mathrm{S} 1 b$ & 2.2909 (16) \\
\hline $\mathrm{Fe}-\mathrm{S} 3$ & $2.3159(15)$ & $\mathrm{Fe} 2 b-\mathrm{S} 3 b$ & $2.3029(15)$ \\
\hline $\mathrm{O} 1-\mathrm{C} 34$ & $1.065(7)$ & $\mathrm{O} 1 b-\mathrm{C} 34 b$ & $0.861(8)$ \\
\hline $\mathrm{O} 2-\mathrm{C} 35$ & $1.151(7)$ & $\mathrm{O} 2 b-\mathrm{C} 35 b$ & $1.137(7)$ \\
\hline $\mathrm{P} 1-\mathrm{Ni}-\mathrm{P} 2$ & $92.88(5)$ & $\mathrm{O} 2-\mathrm{C} 35-\mathrm{Fe}$ & $176.9(5)$ \\
\hline $\mathrm{P} 1-\mathrm{Ni}-\mathrm{S} 3$ & $165.43(6)$ & $\mathrm{P} 1 b-\mathrm{Ni} 1 b-\mathrm{P} 2 b$ & $92.84(6)$ \\
\hline $\mathrm{P} 2-\mathrm{Ni}-\mathrm{S} 3$ & $88.90(5)$ & $\mathrm{P} 1 b-\mathrm{Ni} 1 b-\mathrm{S} 1 b$ & $87.92(6)$ \\
\hline $\mathrm{P} 1-\mathrm{Ni}-\mathrm{S} 1$ & $87.87(5)$ & $\mathrm{P} 2 b-\mathrm{Ni} 1 b-\mathrm{S} 1 b$ & $161.55(6)$ \\
\hline $\mathrm{P} 2-\mathrm{Ni}-\mathrm{S} 1$ & $160.38(6)$ & $\mathrm{P} 1 b-\mathrm{Ni} 1 b-\mathrm{S} 3 b$ & $164.85(6)$ \\
\hline $\mathrm{S} 3-\mathrm{Ni}-\mathrm{S} 1$ & $85.67(5)$ & $\mathrm{P} 2 b-\mathrm{Ni} 1 b-\mathrm{S} 3 b$ & $88.78(5)$ \\
\hline $\mathrm{P} 1-\mathrm{Ni}-\mathrm{Cl}$ & $88.51(5)$ & $\mathrm{S} 1 b-\mathrm{Ni} 1 b-\mathrm{S} 3 b$ & $85.85(5)$ \\
\hline $\mathrm{P} 2-\mathrm{Ni}-\mathrm{Cl}$ & $92.94(5)$ & $\mathrm{P} 1 b-\mathrm{Ni} 1 b-\mathrm{Cl} 1 b$ & $88.79(5)$ \\
\hline $\mathrm{S} 3-\mathrm{Ni}-\mathrm{Cl}$ & $105.85(5)$ & $\mathrm{P} 2 b-\mathrm{Ni} 1 b-\mathrm{C} 11 b$ & $92.29(5)$ \\
\hline $\mathrm{S} 1-\mathrm{Ni}-\mathrm{Cl}$ & $106.68(5)$ & $\mathrm{S} 1 b-\mathrm{Ni} 1 b-\mathrm{Cl} 1 b$ & $106.16(6)$ \\
\hline $\mathrm{C} 35-\mathrm{Fe}-\mathrm{C} 34$ & $91.6(2)$ & $\mathrm{S} 3 b-\mathrm{Ni} 1 b-\mathrm{Cl} 1 b$ & $106.21(5)$ \\
\hline $\mathrm{C} 35-\mathrm{Fe}-\mathrm{N}$ & $175.2(2)$ & $\mathrm{C} 35 b-\mathrm{Fe} 2 b-\mathrm{C} 34 b$ & $90.1(3)$ \\
\hline $\mathrm{C} 34-\mathrm{Fe}-\mathrm{N}$ & $92.5(2)$ & $\mathrm{C} 35 b-\mathrm{Fe} 2 b-\mathrm{N} 1 b$ & $177.9(2)$ \\
\hline $\mathrm{C} 35-\mathrm{Fe}-\mathrm{S} 1$ & $95.72(19)$ & $\mathrm{C} 34 b-\mathrm{Fe} 2 b-\mathrm{N} 1 b$ & $91.8(2)$ \\
\hline $\mathrm{C} 34-\mathrm{Fe}-\mathrm{S} 1$ & $96.10(18)$ & $\mathrm{C} 35 b-\mathrm{Fe} 2 b-\mathrm{S} 2 b$ & 94.41 (19) \\
\hline $\mathrm{N}-\mathrm{Fe}-\mathrm{S} 1$ & $86.26(12)$ & $\mathrm{C} 34 b-\mathrm{Fe} 2 b-\mathrm{S} 2 b$ & $87.86(18)$ \\
\hline $\mathrm{C} 35-\mathrm{Fe}-\mathrm{S} 2$ & 90.91 (19) & $\mathrm{N} 1 b-\mathrm{Fe} 2 b-\mathrm{S} 2 b$ & $86.57(13)$ \\
\hline $\mathrm{C} 34-\mathrm{Fe}-\mathrm{S} 2$ & $87.63(18)$ & $\mathrm{C} 35 b-\mathrm{Fe} 2 b-\mathrm{S} 1 b$ & $92.44(18)$ \\
\hline $\mathrm{N}-\mathrm{Fe}-\mathrm{S} 2$ & $86.83(12)$ & $\mathrm{C} 34 b-\mathrm{Fe} 2 b-\mathrm{S} 1 b$ & 95.97 (18) \\
\hline $\mathrm{S} 1-\mathrm{Fe}-\mathrm{S} 2$ & $172.29(7)$ & $\mathrm{N} 1 b-\mathrm{Fe} 2 b-\mathrm{S} 1 b$ & $86.47(13)$ \\
\hline $\mathrm{C} 35-\mathrm{Fe}-\mathrm{S} 3$ & $88.34(18)$ & $\mathrm{S} 2 b-\mathrm{Fe} 2 b-\mathrm{S} 1 b$ & $172.15(7)$ \\
\hline $\mathrm{C} 34-\mathrm{Fe}-\mathrm{S} 3$ & $179.60(19)$ & $\mathrm{C} 35 b-\mathrm{Fe} 2 b-\mathrm{S} 3 b$ & 90.78 (19) \\
\hline $\mathrm{N}-\mathrm{Fe}-\mathrm{S} 3$ & $87.58(12)$ & $\mathrm{C} 34 b-\mathrm{Fe} 2 b-\mathrm{S} 3 b$ & $179.1(2)$ \\
\hline $\mathrm{S} 1-\mathrm{Fe}-\mathrm{S} 3$ & $83.53(5)$ & $\mathrm{N} 1 b-\mathrm{Fe} 2 b-\mathrm{S} 3 b$ & $87.29(13)$ \\
\hline $\mathrm{S} 2-\mathrm{Fe}-\mathrm{S} 3$ & $92.76(6)$ & $\mathrm{S} 2 b-\mathrm{Fe} 2 b-\mathrm{S} 3 b$ & $92.30(6)$ \\
\hline $\mathrm{Ni}-\mathrm{S} 1-\mathrm{Fe}$ & $95.51(6)$ & $\mathrm{S} 1 b-\mathrm{Fe} 2 b-\mathrm{S} 3 b$ & $83.76(5)$ \\
\hline $\mathrm{O} 1-\mathrm{C} 34-\mathrm{Fe}$ & $175.3(5)$ & $\mathrm{Ni} 1 B-\mathrm{S} 1 B-\mathrm{Fe} 2 B$ & $95.18(6)$ \\
\hline $\mathrm{Fe}-\mathrm{S} 1-\mathrm{C} 29-\mathrm{C} 28$ & $-9.4(6)$ & $\mathrm{C} 29 b-\mathrm{C} 28 b-\mathrm{N} 1 b-\mathrm{Fe} 2 b$ & $-43.0(10)$ \\
\hline $\mathrm{S} 1-\mathrm{C} 29-\mathrm{C} 28-\mathrm{N}$ & $38.6(8)$ & $\mathrm{Fe} 2 b-\mathrm{S} 2 b-\mathrm{C} 31 b-\mathrm{C} 30 b$ & $25.8(8)$ \\
\hline $\mathrm{C} 29-\mathrm{C} 28-\mathrm{N}-\mathrm{Fe}$ & $-51.9(7)$ & $\mathrm{S} 2 b-\mathrm{C} 31 b-\mathrm{C} 30 b-\mathrm{N} 1 b$ & $-54.9(10)$ \\
\hline $\mathrm{Fe}-\mathrm{S} 2-\mathrm{C} 31-\mathrm{C} 30$ & $23.7(6)$ & $\mathrm{C} 31 b-\mathrm{C} 30 b-\mathrm{N} 1 b-\mathrm{Fe} 2 b$ & $58.7(9)$ \\
\hline $\mathrm{S} 2-\mathrm{C} 31-\mathrm{C} 30-\mathrm{N}$ & $-51.7(8)$ & $\mathrm{Fe} 2 b-\mathrm{S} 3 b-\mathrm{C} 33 b-\mathrm{C} 32 b$ & $-35.8(7)$ \\
\hline $\mathrm{C} 31-\mathrm{C} 30-\mathrm{N}-\mathrm{Fe}$ & $55.3(7)$ & $\mathrm{S} 3 b-\mathrm{C} 33 b-\mathrm{C} 32 b-\mathrm{N} 1 b$ & $42.1(10)$ \\
\hline $\mathrm{Fe}-\mathrm{S} 3-\mathrm{C} 33-\mathrm{C} 32$ & $-38.3(6)$ & $\mathrm{C} 33 b-\mathrm{C} 32 b-\mathrm{N} 1 b-\mathrm{Fe} 2 b$ & $-24.5(10)$ \\
\hline $\mathrm{S} 3-\mathrm{C} 33-\mathrm{C} 32-\mathrm{N}$ & $45.8(8)$ & $\mathrm{P} 1-\mathrm{C} 1-\mathrm{C} 2-\mathrm{C} 3$ & $-68.5(8)$ \\
\hline $\mathrm{C} 33-\mathrm{C} 32-\mathrm{N}-\mathrm{Fe}$ & $-27.3(8)$ & $\mathrm{C} 1-\mathrm{C} 2-\mathrm{C} 3-\mathrm{P} 2$ & $69.7(8)$ \\
\hline $\mathrm{Fe} 2 b-\mathrm{S} 1 b-\mathrm{C} 29 b-\mathrm{C} 28 b$ & $-3.0(8)$ & $\mathrm{P} 1 b-\mathrm{C} 1 b-\mathrm{C} 2 b-\mathrm{C} 3 b$ & $-69.3(8)$ \\
\hline $\mathrm{S} 1 b-\mathrm{C} 29 b-\mathrm{C} 28 b-\mathrm{N} 1 b$ & $29.6(12)$ & $\mathrm{C} 1 b-\mathrm{C} 2 b-\mathrm{C} 3 b-\mathrm{P} 2 b$ & $67.1(8)$ \\
\hline
\end{tabular}

$\mathrm{H}$ atoms were not located for the $\mathrm{CH}_{3} \mathrm{CN}$ solvent molecules. The value of the Flack (1983) parameter indicates an inversion twin. All other $\mathrm{H}$ atoms were positioned geometrically $(\mathrm{C}-\mathrm{H}=0.95-0.99 \AA)$ and refined as riding $\left[U_{\text {iso }}(\mathrm{H})=1.2 U_{\text {eq }}(\mathrm{C})\right]$.

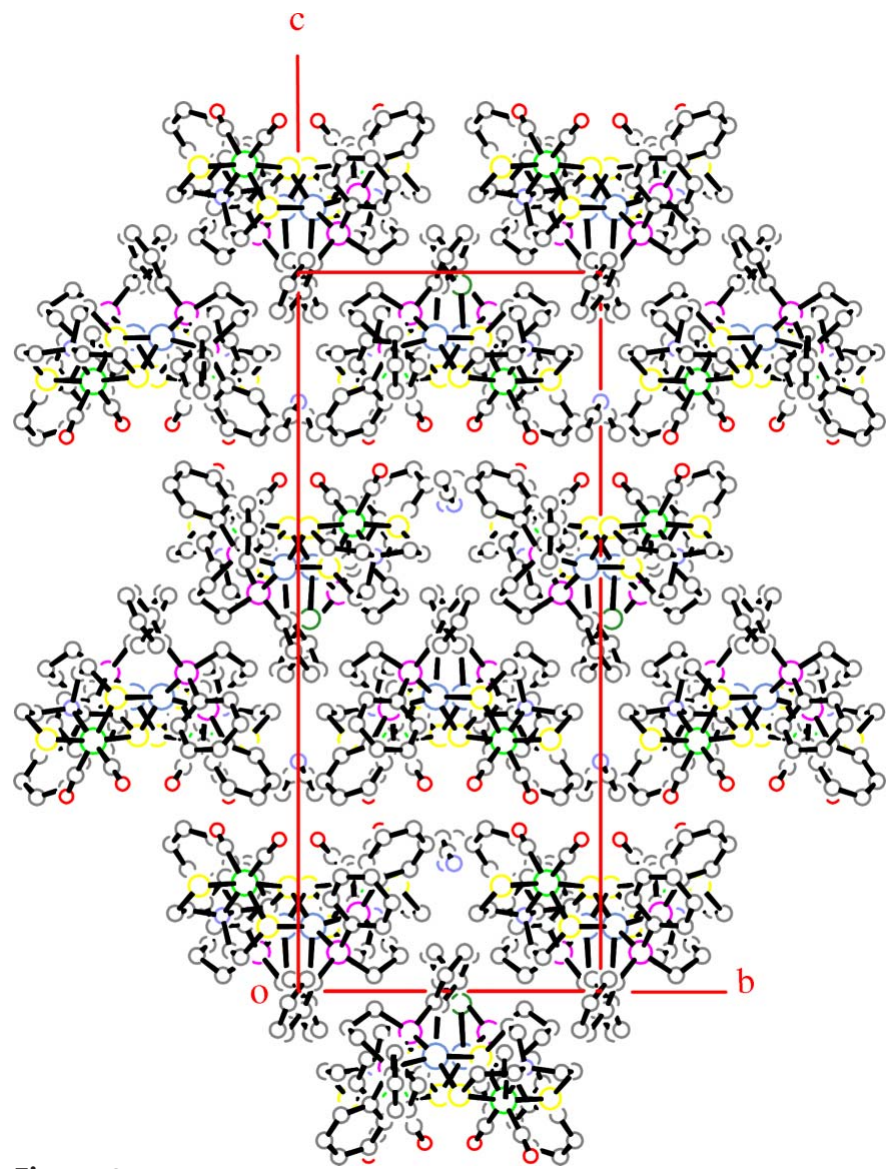

Figure 3

The packing, viewed along the crystallographic $a$ axis. Atoms are represented by arbitrary spheres. $\mathrm{H}$ atoms have been omitted.

Data collection: COLLECT (Nonius, 2000); cell refinement: $H K L$ $S C A L E P A C K$ (Otwinowski \& Minor, 1997); data reduction: $H K L$ DENZO (Otwinowski \& Minor, 1997) and SCALEPACK; program(s) used to solve structure: SHELXS97 (Sheldrick, 1997); program(s) used to refine structure: SHELXL97 (Sheldrick, 1997); molecular graphics: ORTEP-3 for Windows (Farrugia, 1997); software used to prepare material for publication: WinGX (Farrugia, 1999).

The authors thank the Biotechnology and Biological Sciences Research Council and the John Innes Foundation (SED) for financial support.

\section{References}

Blessing, R. H. (1995). Acta Cryst. A51, 33-38.

Davies, S. C., Evans, D. J., Hughes, D. L., Longhurst, S. \& Sanders, J. R. (1999). Chem. Commun. pp. 1935-1936.

Evans, D. J. \& Pickett, C. J. (2003). Chem. Soc. Rev. 32, 268-275.

Farrugia, L. J. (1997). J. Appl. Cryst. 30, 565.

Farrugia, L. J. (1999). J. Appl. Cryst. 32, 837-838.

Flack, H. D. (1983). Acta Cryst. A39, 876-881.

Nonius (2000). COLLECT. Nonius BV, Delft, The Netherlands.

Otwinowski, Z. \& Minor, W. (1997). Methods in Enzymology, Vol. 276, Macromolecular Crystallography, Part A, edited by C. W. Carter Jr \& R. M. Sweet, pp. 307-326. New York: Academic Press.

Sheldrick, G. M. (1997). SHELXS97 and SHELXL97. University of Göttingen, Germany. 


\section{metal-organic papers}

Smith, M. C., Barclay, J. E., Cramer, S. P., Davies, S. C., Gu, W.-W., Hughes, D. L., Longhurst, S. \& Evans, D. J. (2002). J. Chem. Soc. Dalton Trans. pp. 2641-2647.
Smith, M. C., Barclay, J. E., Davies, S. C., Hughes, D. L. \& Evans, D. J. (2003). Dalton Trans. pp. 4147-4151. 


\section{supporting information}

Acta Cryst. (2005). E61, m1316-m1319 [https://doi.org/10.1107/S1600536805018088]

1,3-Bis(diphenylphosphino) propane- $2 \kappa^{2} P, P^{\prime}$-dicarbonyl- $1 \kappa^{2} C$ -

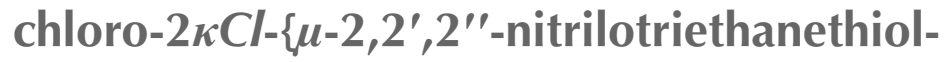

ato(3-)- $1 \kappa^{4} N, S, S^{\prime}, S^{\prime \prime}: 2 \kappa^{2} S, S^{\prime}$ \}iron(II)nickel(II) acetonitrile solvate

S. E. Duff, P. B. Hitchcock, S. C. Davies, J. E. Barclay and D. J. Evans

(I)

Crystal data

$\left[\mathrm{Fe}\left(\mathrm{C}_{6} \mathrm{H}_{12} \mathrm{NS}_{3}\right)(\mathrm{CO})_{2} \mathrm{NiCl}\left(\mathrm{C}_{27} \mathrm{H}_{26} \mathrm{P}_{2}\right)\right] \cdot \mathrm{C}_{2} \mathrm{H}_{3} \mathrm{~N}$

$M_{r}=853.85$

Orthorhombic, $P c a 2_{1}$

Hall symbol: P 2c -2ac

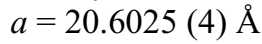

$b=12.4769(2) \AA$

$c=29.7090(6) \AA$

$V=7636.9(2) \AA^{3}$

$Z=8$

$F(000)=3536$

$D_{\mathrm{x}}=1.485 \mathrm{Mg} \mathrm{m}^{-3}$

Mo $K \alpha$ radiation, $\lambda=0.71073 \AA$

Cell parameters from 117428 reflections

$\theta=3.7-23.8^{\circ}$

$\mu=1.23 \mathrm{~mm}^{-1}$

$T=173 \mathrm{~K}$

Plate, brown

$0.2 \times 0.2 \times 0.02 \mathrm{~mm}$

Data collection

Nonius KappaCCD diffractometer

Radiation source: Enraf-Nonius FR590

Graphite monochromator

Detector resolution: 9 pixels $\mathrm{mm}^{-1}$

$\varphi$ or $\omega$ ? scans

Absorption correction: multi-scan (Blessing, 1995)

$T_{\min }=0.823, T_{\max }=0.979$

\section{Refinement}

Refinement on $F^{2}$

Least-squares matrix: full

$R\left[F^{2}>2 \sigma\left(F^{2}\right)\right]=0.052$

$w R\left(F^{2}\right)=0.107$

$S=1.07$

10246 reflections

884 parameters

1 restraint

Primary atom site location: structure-invariant direct methods

Secondary atom site location: difference Fourier map
24708 measured reflections 10246 independent reflections 8837 reflections with $I>2 \sigma(I)$

$R_{\text {int }}=0.052$

$\theta_{\text {max }}=23.7^{\circ}, \theta_{\text {min }}=3.8^{\circ}$

$h=-22 \rightarrow 22$

$k=-14 \rightarrow 13$

$l=-27 \rightarrow 33$

Hydrogen site location: inferred from neighbouring sites

$\mathrm{H}$-atom parameters constrained

$w=1 /\left[\sigma^{2}\left(F_{\mathrm{o}}^{2}\right)+30.7123 P\right]$

where $P=\left(F_{\mathrm{o}}^{2}+2 F_{\mathrm{c}}{ }^{2}\right) / 3$

$(\Delta / \sigma)_{\max }=0.006$

$\Delta \rho_{\max }=0.88 \mathrm{e} \AA^{-3}$

$\Delta \rho_{\min }=-0.63$ e $\AA^{-3}$

Absolute structure: Flack (1983), 4317 Friedel pairs?

Absolute structure parameter: 0.604 (14) 


\section{Special details}

Geometry. All e.s.d.'s (except the e.s.d. in the dihedral angle between two 1.s. planes) are estimated using the full covariance matrix. The cell e.s.d.'s are taken into account individually in the estimation of e.s.d.'s in distances, angles and torsion angles; correlations between e.s.d.'s in cell parameters are only used when they are defined by crystal symmetry. An approximate (isotropic) treatment of cell e.s.d.'s is used for estimating e.s.d.'s involving 1.s. planes.

Refinement. $\mathrm{H}$ atoms were omitted for the $\mathrm{CH} 3 \mathrm{CN}$ solvate molecules.

Refinement of $F^{2}$ against ALL reflections. The weighted $R$-factor $w R$ and goodness of fit $S$ are based on $F^{2}$, conventional $R$-factors $R$ are based on $F$, with $F$ set to zero for negative $F^{2}$. The threshold expression of $F^{2}>\sigma\left(F^{2}\right)$ is used only for calculating $R$-factors(gt) etc. and is not relevant to the choice of reflections for refinement. $R$-factors based on $F^{2}$ are statistically about twice as large as those based on $F$, and $R$ - factors based on ALL data will be even larger.

Fractional atomic coordinates and isotropic or equivalent isotropic displacement parameters $\left(\AA^{2}\right)$

\begin{tabular}{|c|c|c|c|c|}
\hline & $x$ & $y$ & $z$ & $U_{\text {iso }} * / U_{\text {eq }}$ \\
\hline $\mathrm{Ni}$ & $0.31580(3)$ & $0.45332(5)$ & $-0.09044(2)$ & $0.01991(16)$ \\
\hline $\mathrm{Fe}$ & $0.31008(4)$ & $0.67952(5)$ & $-0.15192(2)$ & $0.02202(19)$ \\
\hline $\mathrm{Cl}$ & $0.38331(6)$ & $0.46280(10)$ & $-0.01746(4)$ & $0.0283(3)$ \\
\hline $\mathrm{S} 1$ & $0.37554(7)$ & $0.53252(10)$ & $-0.14477(5)$ & $0.0261(4)$ \\
\hline $\mathrm{S} 2$ & $0.24863(7)$ & $0.83311(11)$ & $-0.14947(6)$ & $0.0371(4)$ \\
\hline S3 & $0.25547(6)$ & $0.60378(9)$ & $-0.09167(5)$ & $0.0222(3)$ \\
\hline $\mathrm{P} 1$ & $0.36558(6)$ & $0.30219(10)$ & $-0.10683(5)$ & 0.0213 \\
\hline $\mathrm{P} 2$ & $0.23460(7)$ & $0.37016(10)$ & $-0.05694(5)$ & $0.0206(3)$ \\
\hline $\mathrm{O} 1$ & $0.3765(2)$ & $0.7682(4)$ & $-0.22910(16)$ & $0.0607(15)$ \\
\hline $\mathrm{O} 2$ & $0.2146(2)$ & $0.5822(4)$ & $-0.21136(14)$ & $0.0522(13)$ \\
\hline $\mathrm{N}$ & $0.3700(2)$ & $0.7517(3)$ & $-0.10603(14)$ & $0.0237(12)$ \\
\hline $\mathrm{C} 1$ & $0.3604(3)$ & $0.1952(4)$ & $-0.06428(18)$ & $0.0283(15)$ \\
\hline $\mathrm{H} 1 \mathrm{~A}$ & 0.383 & 0.131 & -0.0759 & $0.034^{*}$ \\
\hline H1B & 0.3837 & 0.219 & -0.0369 & $0.034^{*}$ \\
\hline $\mathrm{C} 2$ & $0.2923(2)$ & $0.1643(4)$ & $-0.05141(18)$ & $0.0221(13)$ \\
\hline $\mathrm{H} 2 \mathrm{~A}$ & 0.2939 & 0.0985 & -0.0328 & $0.027 *$ \\
\hline $\mathrm{H} 2 \mathrm{~B}$ & 0.2676 & 0.1475 & -0.0791 & $0.027^{*}$ \\
\hline $\mathrm{C} 3$ & $0.2562(3)$ & $0.2519(4)$ & $-0.02522(18)$ & $0.0247(14)$ \\
\hline $\mathrm{H} 3 \mathrm{~A}$ & 0.2837 & 0.2741 & 0.0005 & $0.03 *$ \\
\hline $\mathrm{H} 3 \mathrm{~B}$ & 0.216 & 0.2204 & -0.0127 & $0.03 *$ \\
\hline $\mathrm{C} 4$ & $0.3346(2)$ & $0.2377(4)$ & $-0.15762(18)$ & $0.0233(14)$ \\
\hline $\mathrm{C} 5$ & $0.3599(3)$ & $0.1412(4)$ & -0.17244 (19) & $0.0338(16)$ \\
\hline H5 & 0.3976 & 0.1126 & -0.1585 & $0.041 *$ \\
\hline C6 & $0.3306(3)$ & $0.0856(5)$ & -0.2077 (2) & $0.0453(19)$ \\
\hline H6 & 0.3457 & 0.0166 & -0.2163 & $0.054 *$ \\
\hline $\mathrm{C} 7$ & 0.2780 & $0.1339(6)$ & $-0.2302(2)$ & 0.0494 (19) \\
\hline $\mathrm{H} 7$ & 0.2583 & 0.0982 & -0.2549 & $0.059 *$ \\
\hline $\mathrm{C} 8$ & 0.2550 & $0.2326(6)$ & $-0.2168(2)$ & $0.047(2)$ \\
\hline H8 & 0.2193 & 0.2644 & -0.232 & $0.057^{*}$ \\
\hline $\mathrm{C} 9$ & 0.2838 & $0.2843(5)$ & $-0.18156(18)$ & $0.0355(17)$ \\
\hline H9 & 0.2688 & 0.3535 & -0.1732 & $0.043 *$ \\
\hline $\mathrm{C} 10$ & $0.4536(2)$ & $0.3113(4)$ & $-0.11620(17)$ & $0.0209(13)$ \\
\hline $\mathrm{C} 11$ & $0.4951(3)$ & $0.3159(4)$ & $-0.0793(2)$ & $0.0334(16)$ \\
\hline H11 & 0.478 & 0.3175 & -0.0496 & $0.04^{*}$ \\
\hline
\end{tabular}




\begin{tabular}{|c|c|c|c|c|}
\hline $\mathrm{C} 12$ & $0.5619(3)$ & $0.3182(4)$ & $-0.0861(2)$ & $0.0355(16)$ \\
\hline H12 & 0.5902 & 0.3182 & -0.0609 & $0.043^{*}$ \\
\hline $\mathrm{C} 13$ & $0.5875(3)$ & $0.3203(4)$ & $-0.1287(2)$ & $0.0394(17)$ \\
\hline H13 & 0.6332 & 0.3213 & -0.1329 & $0.047 *$ \\
\hline $\mathrm{C} 14$ & $0.5468(3)$ & $0.3209(5)$ & $-0.1650(2)$ & $0.0428(18)$ \\
\hline H14 & 0.5643 & 0.3251 & -0.1946 & $0.051^{*}$ \\
\hline $\mathrm{C} 15$ & $0.4797(3)$ & $0.3155(4)$ & $-0.15904(19)$ & $0.0319(16)$ \\
\hline H15 & 0.4518 & 0.3148 & -0.1845 & $0.038^{*}$ \\
\hline C16 & $0.1716(2)$ & $0.3275(4)$ & $-0.09666(17)$ & $0.0214(13)$ \\
\hline $\mathrm{C} 17$ & $0.1544(3)$ & $0.3955(4)$ & $-0.13105(18)$ & $0.0272(15)$ \\
\hline H17 & 0.1719 & 0.4659 & -0.1318 & $0.033^{*}$ \\
\hline $\mathrm{C} 18$ & 0.1117 (3) & $0.3632(5)$ & $-0.16473(19)$ & $0.0321(16)$ \\
\hline H18 & 0.1003 & 0.4109 & -0.1884 & $0.039 *$ \\
\hline C19 & $0.0864(3)$ & $0.2615(5)$ & $-0.1633(2)$ & $0.0371(17)$ \\
\hline H19 & 0.0582 & 0.2382 & -0.1866 & $0.045^{*}$ \\
\hline $\mathrm{C} 20$ & $0.1014(3)$ & $0.1927(5)$ & -0.12845 (19) & $0.0340(16)$ \\
\hline $\mathrm{H} 20$ & 0.0829 & 0.1231 & -0.1273 & $0.041 *$ \\
\hline $\mathrm{C} 21$ & $0.1441(3)$ & $0.2265(4)$ & $-0.0948(2)$ & $0.0303(15)$ \\
\hline $\mathrm{H} 21$ & 0.1544 & 0.1799 & -0.0705 & $0.036^{*}$ \\
\hline $\mathrm{C} 22$ & 0.1909 (3) & $0.4486(4)$ & $-0.01484(18)$ & $0.0252(14)$ \\
\hline $\mathrm{C} 23$ & $0.1256(3)$ & $0.4710(4)$ & $-0.01890(19)$ & $0.0320(15)$ \\
\hline $\mathrm{H} 23$ & 0.1022 & 0.4454 & -0.0443 & $0.038^{*}$ \\
\hline $\mathrm{C} 24$ & $0.0935(3)$ & $0.5310(4)$ & $0.0139(2)$ & $0.0384(17)$ \\
\hline $\mathrm{H} 24$ & 0.0483 & 0.5449 & 0.0112 & $0.046^{*}$ \\
\hline $\mathrm{C} 25$ & $0.1277(3)$ & $0.5698(4)$ & $0.0500(2)$ & $0.0411(17)$ \\
\hline $\mathrm{H} 25$ & 0.106 & 0.6114 & 0.0722 & $0.049^{*}$ \\
\hline $\mathrm{C} 26$ & $0.1925(3)$ & $0.5492(4)$ & $0.0544(2)$ & $0.0360(16)$ \\
\hline H26 & 0.2158 & 0.5771 & 0.0794 & $0.043^{*}$ \\
\hline $\mathrm{C} 27$ & $0.2246(3)$ & $0.4872(4)$ & $0.02205(19)$ & $0.0317(16)$ \\
\hline $\mathrm{H} 27$ & 0.2694 & 0.4716 & 0.0254 & $0.038^{*}$ \\
\hline $\mathrm{C} 28$ & $0.4397(3)$ & $0.7145(4)$ & $-0.1139(2)$ & $0.0360(17)$ \\
\hline $\mathrm{H} 28 \mathrm{~A}$ & 0.4557 & 0.7448 & -0.1426 & $0.043 *$ \\
\hline $\mathrm{H} 28 \mathrm{~B}$ & 0.4677 & 0.7422 & -0.0894 & $0.043^{*}$ \\
\hline C29 & $0.4449(3)$ & $0.5936(4)$ & $-0.1155(2)$ & $0.0355(17)$ \\
\hline $\mathrm{H} 29 \mathrm{~A}$ & 0.4471 & 0.5653 & -0.0844 & $0.043 *$ \\
\hline H29B & 0.4855 & 0.5733 & -0.1311 & $0.043^{*}$ \\
\hline $\mathrm{C} 30$ & $0.3694(3)$ & $0.8705(4)$ & $-0.1134(2)$ & $0.0317(16)$ \\
\hline $\mathrm{H} 30 \mathrm{~A}$ & 0.3886 & 0.8875 & -0.143 & $0.038^{*}$ \\
\hline $\mathrm{H} 30 \mathrm{~B}$ & 0.3957 & 0.9062 & -0.0899 & $0.038^{*}$ \\
\hline C31 & $0.2998(3)$ & $0.9119(4)$ & $-0.1115(2)$ & $0.0341(16)$ \\
\hline H31A & 0.2987 & 0.9883 & -0.1205 & $0.041 *$ \\
\hline H31B & 0.2831 & 0.9063 & -0.0803 & $0.041 *$ \\
\hline C32 & $0.3548(3)$ & $0.7250(4)$ & $-0.0577(2)$ & $0.0348(16)$ \\
\hline $\mathrm{H} 32 \mathrm{~A}$ & 0.3664 & 0.7873 & -0.0386 & $0.042 *$ \\
\hline H32B & 0.3823 & 0.664 & -0.0482 & $0.042 *$ \\
\hline C33 & $0.2844(3)$ & $0.6965(4)$ & $-0.04927(19)$ & $0.0273(15)$ \\
\hline $\mathrm{H} 33 \mathrm{~A}$ & 0.2577 & 0.7625 & -0.0499 & $0.033^{*}$ \\
\hline H33B & 0.28 & 0.6636 & -0.0191 & $0.033^{*}$ \\
\hline
\end{tabular}




\begin{tabular}{|c|c|c|c|c|}
\hline C34 & $0.3534(3)$ & $0.7384(4)$ & $-0.19960(18)$ & $0.0273(15)$ \\
\hline $\mathrm{C} 35$ & $0.2534(3)$ & $0.6183(5)$ & $-0.18797(18)$ & $0.0310(16)$ \\
\hline NilB & $0.44894(3)$ & $-0.04767(5)$ & $0.08998(2)$ & $0.02046(17)$ \\
\hline $\mathrm{Fe} 2 \mathrm{~B}$ & 0.45935 (4) & $0.17831(6)$ & $0.15060(3)$ & $0.0243(2)$ \\
\hline Cl1B & $0.37760(7)$ & $-0.03749(11)$ & $0.01804(5)$ & $0.0343(4)$ \\
\hline S1B & $0.39179(7)$ & $0.03313(11)$ & $0.14485(5)$ & 0.0295 (4) \\
\hline S2B & $0.52230(9)$ & $0.32868(14)$ & $0.14654(7)$ & $0.0539(5)$ \\
\hline S3B & $0.51087(6)$ & $0.10121(10)$ & $0.08990(5)$ & $0.0217(3)$ \\
\hline P1B & $0.39939(7)$ & $-0.19807(11)$ & $0.10794(5)$ & 0.0240 (4) \\
\hline $\mathrm{P} 2 \mathrm{~B}$ & $0.52737(7)$ & $-0.13218(10)$ & $0.05408(5)$ & $0.0214(4)$ \\
\hline O1B & $0.3979(3)$ & $0.2697(4)$ & $0.2241(2)$ & $0.0724(18)$ \\
\hline $\mathrm{O} 2 \mathrm{~B}$ & $0.5449(2)$ & $0.0636(3)$ & $0.21111(14)$ & $0.0466(13)$ \\
\hline N1B & $0.3986(2)$ & $0.2527(3)$ & $0.10496(15)$ & $0.0293(13)$ \\
\hline $\mathrm{C} 1 \mathrm{~B}$ & $0.3990(3)$ & $-0.3008(4)$ & $0.06485(18)$ & $0.0254(14)$ \\
\hline H1B1 & 0.3757 & -0.3643 & 0.0766 & $0.03 *$ \\
\hline H1B2 & 0.3745 & -0.2738 & 0.0385 & $0.03^{*}$ \\
\hline $\mathrm{C} 2 \mathrm{~B}$ & $0.4664(3)$ & $-0.3360(4)$ & 0.04915 (19) & $0.0280(15)$ \\
\hline H2B1 & 0.4618 & -0.401 & 0.0303 & $0.034 *$ \\
\hline H2B2 & 0.4925 & -0.3557 & 0.0759 & $0.034^{*}$ \\
\hline $\mathrm{C} 3 \mathrm{~B}$ & $0.5039(3)$ & $-0.2500(4)$ & $0.02196(18)$ & $0.0226(14)$ \\
\hline H3B1 & 0.4766 & -0.2268 & -0.0036 & $0.027^{*}$ \\
\hline H3B2 & 0.5436 & -0.2833 & 0.0093 & $0.027 *$ \\
\hline C4B & $0.4336(2)$ & $-0.2665(4)$ & $0.15613(18)$ & $0.0256(14)$ \\
\hline C5B & $0.4853(3)$ & $-0.2249(5)$ & $0.1791(2)$ & $0.0340(16)$ \\
\hline H5B & 0.5007 & -0.1556 & 0.1712 & $0.041 *$ \\
\hline C6B & $0.5159(3)$ & $-0.2807(5)$ & $0.2136(2)$ & $0.0464(19)$ \\
\hline H6B & 0.5518 & -0.2496 & 0.2288 & $0.056^{*}$ \\
\hline C7B & $0.4944(4)$ & $-0.3808(5)$ & $0.2258(2)$ & $0.048(2)$ \\
\hline H7B & 0.5161 & -0.4209 & 0.2484 & $0.058^{*}$ \\
\hline $\mathrm{C} 8 \mathrm{~B}$ & $0.4400(4)$ & $-0.4215(5)$ & $0.2042(2)$ & $0.049(2)$ \\
\hline H8B & 0.4224 & -0.4883 & 0.2137 & $0.059^{*}$ \\
\hline C9B & $0.4115(3)$ & $-0.3675(5)$ & $0.1698(2)$ & $0.0413(18)$ \\
\hline H9B & 0.3756 & -0.3989 & 0.1547 & $0.05^{*}$ \\
\hline $\mathrm{C} 10 \mathrm{~B}$ & $0.3133(3)$ & $-0.1854(4)$ & $0.1226(2)$ & $0.0365(18)$ \\
\hline C11B & $0.2664(3)$ & $-0.1792(5)$ & $0.0880(3)$ & $0.0470(19)$ \\
\hline H11B & 0.2796 & -0.1817 & 0.0574 & $0.056^{*}$ \\
\hline $\mathrm{C} 12 \mathrm{~B}$ & $0.2013(3)$ & $-0.1696(5)$ & $0.0986(3)$ & $0.060(2)$ \\
\hline H12B & 0.1695 & -0.1667 & 0.0754 & $0.072 *$ \\
\hline C13B & 0.1832 (4) & $-0.1644(5)$ & 0.1428 (3) & 0.070 \\
\hline H13B & 0.1384 & -0.1575 & 0.1498 & $0.084 *$ \\
\hline C14B & $0.2272(3)$ & $-0.1688(5)$ & $0.1773(3)$ & $0.064(2)$ \\
\hline H14B & 0.2128 & -0.1658 & 0.2077 & $0.076^{*}$ \\
\hline C15B & 0.2949 (3) & $-0.1780(5)$ & $0.1674(2)$ & $0.049(2)$ \\
\hline H15B & 0.3264 & -0.1791 & 0.1908 & $0.059 *$ \\
\hline $\mathrm{C} 16 \mathrm{~B}$ & $0.5912(2)$ & $-0.1767(4)$ & $0.09261(17)$ & $0.0213(13)$ \\
\hline C17B & $0.6102(3)$ & $-0.1082(4)$ & $0.12771(18)$ & $0.0266(15)$ \\
\hline H17B & 0.5926 & -0.0379 & 0.1294 & $0.032 *$ \\
\hline C18B & $0.6529(3)$ & $-0.1410(4)$ & $0.1590(2)$ & $0.0328(16)$ \\
\hline
\end{tabular}




\begin{tabular}{|c|c|c|c|c|}
\hline H18B & 0.6654 & -0.0928 & 0.1822 & $0.039 *$ \\
\hline C19B & $0.6788(3)$ & $-0.2411(5)$ & $0.1584(2)$ & $0.0325(16)$ \\
\hline H19B & 0.7071 & -0.264 & 0.1817 & $0.039 *$ \\
\hline $\mathrm{C} 20 \mathrm{~B}$ & $0.6634(3)$ & $-0.3097(4)$ & $0.1230(2)$ & $0.0334(16)$ \\
\hline $\mathrm{H} 20 \mathrm{~B}$ & 0.6831 & -0.3784 & 0.1213 & $0.04 *$ \\
\hline $\mathrm{C} 21 \mathrm{~B}$ & $0.6193(3)$ & $-0.2783(4)$ & $0.0903(2)$ & $0.0262(14)$ \\
\hline $\mathrm{H} 21 \mathrm{~B}$ & 0.6084 & -0.3256 & 0.0664 & $0.031 *$ \\
\hline $\mathrm{C} 22 \mathrm{~B}$ & $0.5708(3)$ & $-0.0520(4)$ & 0.01205 (18) & $0.0240(14)$ \\
\hline $\mathrm{C} 23 \mathrm{~B}$ & $0.6360(3)$ & $-0.0269(4)$ & $0.0164(2)$ & $0.0328(16)$ \\
\hline H23B & 0.6597 & -0.0514 & 0.0418 & $0.039^{*}$ \\
\hline $\mathrm{C} 24 \mathrm{~B}$ & $0.6667(3)$ & $0.0341(5)$ & $-0.0165(2)$ & $0.0386(17)$ \\
\hline H24B & 0.7112 & 0.052 & -0.0128 & $0.046^{*}$ \\
\hline $\mathrm{C} 25 \mathrm{~B}$ & $0.6347(3)$ & $0.0686(5)$ & $-0.0536(2)$ & $0.0416(18)$ \\
\hline H25B & 0.6565 & 0.1102 & -0.0758 & $0.05^{*}$ \\
\hline $\mathrm{C} 26 \mathrm{~B}$ & $0.5692(3)$ & $0.0419(5)$ & $-0.0588(2)$ & 0.0451 (19) \\
\hline H26B & 0.5464 & 0.0644 & -0.085 & $0.054 *$ \\
\hline C27B & $0.5377(3)$ & $-0.0163(4)$ & $-0.0265(2)$ & $0.0305(16)$ \\
\hline H27B & 0.4929 & -0.0327 & -0.0301 & $0.037^{*}$ \\
\hline $\mathrm{C} 28 \mathrm{~B}$ & 0.3314 (3) & $0.2115(6)$ & $0.1110(3)$ & $0.064(2)$ \\
\hline $\mathrm{H} 28 \mathrm{C}$ & 0.3121 & 0.2485 & 0.1373 & $0.077^{*}$ \\
\hline $\mathrm{H} 28 \mathrm{D}$ & 0.3058 & 0.2328 & 0.0842 & $0.077^{*}$ \\
\hline C29B & $0.3230(3)$ & 0.0976 (4) & $0.1174(3)$ & $0.045(2)$ \\
\hline $\mathrm{H} 29 \mathrm{C}$ & 0.316 & 0.0634 & 0.0878 & $0.054^{*}$ \\
\hline $\mathrm{H} 29 \mathrm{D}$ & 0.2836 & 0.0854 & 0.1358 & $0.054^{*}$ \\
\hline $\mathrm{C} 30 \mathrm{~B}$ & $0.3981(4)$ & $0.3690(5)$ & $0.1132(3)$ & 0.069 (3) \\
\hline $\mathrm{H} 30 \mathrm{C}$ & 0.3817 & 0.3846 & 0.1438 & $0.082 *$ \\
\hline H30D & 0.3699 & 0.4057 & 0.0911 & $0.082^{*}$ \\
\hline $\mathrm{C} 31 \mathrm{~B}$ & $0.4712(4)$ & $0.4084(5)$ & $0.1082(3)$ & $0.064(2)$ \\
\hline $\mathrm{H} 31 \mathrm{C}$ & 0.4744 & 0.4854 & 0.1159 & $0.076^{*}$ \\
\hline H31D & 0.4859 & 0.3988 & 0.0767 & $0.076^{*}$ \\
\hline C32B & 0.4138 (4) & $0.2251(6)$ & $0.0561(2)$ & $0.064(2)$ \\
\hline $\mathrm{H} 32 \mathrm{C}$ & 0.3842 & 0.1671 & 0.0464 & $0.077^{*}$ \\
\hline H32D & 0.4044 & 0.2887 & 0.0373 & $0.077^{*}$ \\
\hline C33B & $0.4806(3)$ & 0.1911 (4) & $0.04729(18)$ & $0.0264(15)$ \\
\hline $\mathrm{H} 33 \mathrm{C}$ & 0.5089 & 0.2551 & 0.0459 & $0.032 *$ \\
\hline H33D & 0.4825 & 0.155 & 0.0176 & $0.032^{*}$ \\
\hline C34B & $0.4154(3)$ & $0.2430(5)$ & $0.2004(2)$ & $0.0323(17)$ \\
\hline C35B & $0.5116(3)$ & 0.1112 (4) & $0.18801(19)$ & 0.0305 (16) \\
\hline N2 & $0.2291(4)$ & $0.4853(8)$ & $-0.3224(3)$ & $0.160(3)$ \\
\hline N2B & 0.4973 (3) & $0.0005(6)$ & $0.3194(3)$ & 0.093 \\
\hline C36 & $0.3436(4)$ & $0.5235(8)$ & $-0.2875(3)$ & $0.095(3)$ \\
\hline C37 & $0.2791(5)$ & $0.5062(6)$ & $-0.3067(3)$ & $0.081(3)$ \\
\hline $\mathrm{C} 36 \mathrm{~B}$ & $0.3942(4)$ & $-0.0543(9)$ & $0.2759(3)$ & 0.099 (4) \\
\hline C37B & $0.4515(4)$ & $-0.0249(7)$ & 0.3007 (3) & 0.068 (3) \\
\hline
\end{tabular}


Atomic displacement parameters $\left(\AA^{2}\right)$

\begin{tabular}{|c|c|c|c|c|c|c|}
\hline & $U^{11}$ & $U^{22}$ & $U^{33}$ & $U^{12}$ & $U^{13}$ & $U^{23}$ \\
\hline $\mathrm{Ni}$ & $0.0181(3)$ & $0.0166(3)$ & $0.0250(4)$ & $-0.0006(3)$ & -0.0001 & 0.0011 \\
\hline $\mathrm{Fe}$ & $0.0248(4)$ & $0.0188(4)$ & $0.0225(4)$ & 0.0003 (3) & $-0.0007(3)$ & $0.0024(3)$ \\
\hline $\mathrm{Cl}$ & $0.0309(7)$ & $0.0225(6)$ & $0.0316(8)$ & $-0.0039(6)$ & $-0.0071(6)$ & $0.0006(6)$ \\
\hline $\mathrm{S} 1$ & $0.0283(7)$ & $0.0219(7)$ & $0.0282(8)$ & 0.0009 (6) & $0.0052(6)$ & $0.0025(6)$ \\
\hline $\mathrm{S} 2$ & $0.0346(8)$ & $0.0296(7)$ & $0.0470(9)$ & $0.0042(7)$ & $-0.0003(7)$ & $0.0026(7)$ \\
\hline $\mathrm{S} 3$ & $0.0213(6)$ & $0.0170(6)$ & $0.0283(8)$ & $0.0006(6)$ & $0.0024(6)$ & $0.0012(6)$ \\
\hline $\mathrm{P} 1$ & $0.0171(7)$ & $0.0217(7)$ & $0.0250(8)$ & $0.0010(6)$ & $-0.0021(6)$ & $0.0013(6)$ \\
\hline $\mathrm{P} 2$ & $0.0231(7)$ & $0.0165(6)$ & $0.0222(8)$ & $-0.0011(6)$ & $-0.0006(6)$ & $0.0002(6)$ \\
\hline $\mathrm{O} 1$ & $0.072(3)$ & $0.057(3)$ & $0.054(3)$ & $-0.011(3)$ & $-0.001(3)$ & $0.008(2)$ \\
\hline $\mathrm{O} 2$ & 0.049 (3) & $0.068(3)$ & $0.039(3)$ & $-0.015(2)$ & $-0.008(2)$ & $0.002(2)$ \\
\hline $\mathrm{N}$ & $0.031(3)$ & $0.015(2)$ & $0.025(3)$ & $-0.003(2)$ & 0.0064 (19) & $0.0075(18)$ \\
\hline $\mathrm{C} 1$ & $0.040(3)$ & $0.014(3)$ & $0.030(3)$ & $0.000(3)$ & $-0.002(3)$ & $-0.001(2)$ \\
\hline $\mathrm{C} 2$ & $0.020(3)$ & $0.015(2)$ & $0.032(3)$ & $0.001(2)$ & $0.000(2)$ & $-0.004(2)$ \\
\hline $\mathrm{C} 3$ & $0.022(3)$ & $0.025(3)$ & $0.027(3)$ & $-0.002(2)$ & $0.001(2)$ & $0.001(2)$ \\
\hline $\mathrm{C} 4$ & $0.021(3)$ & $0.016(3)$ & $0.033(3)$ & $-0.007(2)$ & $0.005(2)$ & $0.002(2)$ \\
\hline $\mathrm{C} 5$ & $0.043(4)$ & $0.021(3)$ & $0.037(4)$ & 0.007 (3) & $-0.002(3)$ & $0.001(3)$ \\
\hline C6 & $0.069(4)$ & $0.037(3)$ & $0.030(4)$ & $-0.014(3)$ & $0.007(3)$ & $-0.007(3)$ \\
\hline $\mathrm{C} 7$ & $0.045(4)$ & $0.078(5)$ & $0.024(4)$ & $-0.024(4)$ & $-0.001(3)$ & $-0.016(3)$ \\
\hline $\mathrm{C} 8$ & $0.027(3)$ & $0.085(5)$ & $0.030(4)$ & 0.007 (4) & $-0.004(3)$ & -0.009 \\
\hline $\mathrm{C} 9$ & $0.025(3)$ & $0.056(4)$ & $0.026(3)$ & $0.001(3)$ & $0.001(3)$ & $-0.007(3)$ \\
\hline $\mathrm{C} 10$ & 0.027 (3) & $0.011(2)$ & $0.025(3)$ & $0.001(2)$ & $-0.006(2)$ & $-0.001(2)$ \\
\hline C11 & $0.023(3)$ & $0.038(3)$ & $0.039(4)$ & $0.001(3)$ & $-0.004(3)$ & $-0.004(3)$ \\
\hline $\mathrm{C} 12$ & $0.034(3)$ & $0.037(3)$ & $0.036(3)$ & 0.009 (3) & -0.010 & $-0.004(3)$ \\
\hline C13 & $0.018(3)$ & $0.033(3)$ & $0.067(4)$ & $-0.004(3)$ & $-0.013(3)$ & $-0.003(3)$ \\
\hline $\mathrm{C} 14$ & $0.030(3)$ & $0.055(4)$ & $0.043(4)$ & -0.001 & $0.006(3)$ & -0.003 \\
\hline C15 & $0.023(3)$ & $0.043(3)$ & $0.030(3)$ & -0.001 & $-0.009(2)$ & $0.002(3)$ \\
\hline C16 & $0.013(2)$ & $0.024(3)$ & $0.028(3)$ & $-0.014(2)$ & $0.003(2)$ & $-0.006(2)$ \\
\hline C17 & $0.026(3)$ & $0.025(3)$ & $0.031(3)$ & $-0.002(3)$ & $-0.004(2)$ & $0.001(2)$ \\
\hline C18 & $0.029(3)$ & $0.038(3)$ & $0.029(3)$ & $0.003(3)$ & $0.003(3)$ & $0.004(3)$ \\
\hline C19 & $0.024(3)$ & $0.046(4)$ & $0.041(4)$ & $0.000(3)$ & $-0.006(3)$ & -0.005 \\
\hline $\mathrm{C} 20$ & $0.027(3)$ & $0.039(3)$ & $0.036(3)$ & $-0.013(3)$ & $0.000(3)$ & -0.005 \\
\hline $\mathrm{C} 21$ & $0.027(3)$ & $0.027(3)$ & $0.037(3)$ & $-0.003(2)$ & $-0.002(3)$ & $0.004(3)$ \\
\hline $\mathrm{C} 22$ & $0.029(3)$ & $0.015(3)$ & $0.031(3)$ & $-0.005(2)$ & $0.003(3)$ & $0.003(2)$ \\
\hline $\mathrm{C} 23$ & $0.041(3)$ & $0.028(3)$ & $0.027(3)$ & $0.002(3)$ & $0.003(3)$ & $0.000(3)$ \\
\hline $\mathrm{C} 24$ & $0.041(3)$ & $0.032(3)$ & $0.042(4)$ & $-0.003(3)$ & $0.015(3)$ & $0.005(3)$ \\
\hline $\mathrm{C} 25$ & $0.049(4)$ & $0.031(3)$ & $0.044(4)$ & $-0.004(3)$ & $0.023(3)$ & $-0.006(3)$ \\
\hline $\mathrm{C} 26$ & $0.046(4)$ & $0.030(3)$ & $0.032(3)$ & $-0.019(3)$ & $0.008(3)$ & -0.011 \\
\hline $\mathrm{C} 27$ & $0.028(3)$ & $0.038(3)$ & $0.029(3)$ & $-0.007(3)$ & $0.012(3)$ & $0.000(3)$ \\
\hline $\mathrm{C} 28$ & 0.019 (3) & 0.039 (3) & 0.049 (4) & $-0.006(3)$ & $-0.003(3)$ & 0.009 (3) \\
\hline $\mathrm{C} 29$ & $0.022(3)$ & $0.033(3)$ & $0.051(4)$ & -0.003 & $0.007(3)$ & $0.005(3)$ \\
\hline $\mathrm{C} 30$ & $0.033(3)$ & $0.024(3)$ & $0.039(4)$ & $-0.006(3)$ & $-0.004(3)$ & $-0.003(3)$ \\
\hline C31 & $0.043(4)$ & $0.022(3)$ & $0.037(4)$ & 0.007 (3) & $0.002(3)$ & $-0.002(3)$ \\
\hline $\mathrm{C} 32$ & $0.038(3)$ & $0.028(3)$ & $0.039(4)$ & $0.003(3)$ & $-0.006(3)$ & $0.005(3)$ \\
\hline $\mathrm{C} 33$ & $0.027(3)$ & $0.020(3)$ & $0.035(3)$ & $-0.003(2)$ & $0.006(3)$ & $0.009(2)$ \\
\hline C34 & $0.025(3)$ & $0.039(3)$ & $0.018(3)$ & $0.000(3)$ & $0.001(2)$ & $-0.012(2)$ \\
\hline
\end{tabular}




\begin{tabular}{|c|c|c|c|c|c|c|}
\hline C35 & $0.036(3)$ & 0.039 & $0.019(3)$ & $0.002(3)$ & $0.001(3)$ & $0.003(3)$ \\
\hline Ni1B & $0.0192(3)$ & $0.0153(3)$ & 0.0269 (4) & $0.0002(3)$ & $0.0012(3)$ & $0.0002(3)$ \\
\hline $\mathrm{Fe} 2 \mathrm{~B}$ & 0.0289 (4) & 0.0196 (4) & 0.0245 (4) & -0.0009 (4) & $0.0069(3)$ & $0.0001(3)$ \\
\hline Cl1B & $0.0354(8)$ & $0.0289(7)$ & $0.0385(8)$ & $0.0039(7)$ & $-0.0149(6)$ & $-0.0001(7)$ \\
\hline S1B & $0.0301(8)$ & $0.0210(7)$ & $0.0374(9)$ & $-0.0033(6)$ & $0.0135(6)$ & $-0.0024(6)$ \\
\hline S2B & $0.0527(11)$ & $0.0488(10)$ & $0.0602(12)$ & $0.0017(9)$ & $0.0091(9)$ & -0.0036 \\
\hline S3B & $0.0219(7)$ & $0.0193(6)$ & $0.0240(7)$ & 0.0009 (6) & $0.0018(6)$ & $-0.0022(6)$ \\
\hline P1B & $0.0200(7)$ & $0.0178(7)$ & $0.0343(8)$ & $-0.0017(6)$ & $-0.0002(6)$ & $-0.0010(6)$ \\
\hline P2B & $0.0204(7)$ & $0.0197(7)$ & $0.0239(8)$ & $0.0038(6)$ & $-0.0008(6)$ & $-0.0013(6)$ \\
\hline O1B & $0.071(4)$ & $0.060(3)$ & $0.087(4)$ & $-0.010(3)$ & $-0.015(3)$ & $0.033(3)$ \\
\hline $\mathrm{O} 2 \mathrm{~B}$ & $0.054(3)$ & $0.051(3)$ & $0.035(3)$ & $0.000(2)$ & $-0.010(2)$ & $0.000(2)$ \\
\hline N1B & $0.030(3)$ & $0.022(2)$ & $0.036(3)$ & $0.003(2)$ & $0.010(2)$ & $-0.004(2)$ \\
\hline C1B & $0.023(3)$ & $0.026(3)$ & $0.027(3)$ & $-0.005(2)$ & $-0.010(2)$ & $0.000(2)$ \\
\hline $\mathrm{C} 2 \mathrm{~B}$ & $0.037(3)$ & 0.018 (3) & $0.029(3)$ & -0.008 (3) & $-0.006(3)$ & $-0.006(2)$ \\
\hline C3B & $0.027(3)$ & $0.018(3)$ & $0.023(3)$ & $0.002(2)$ & $-0.004(2)$ & $-0.001(2)$ \\
\hline C4B & 0.017 (3) & 0.033 & $0.026(3)$ & $-0.004(3)$ & $0.001(2)$ & $-0.005(3)$ \\
\hline C5B & $0.034(3)$ & $0.030(3)$ & $0.038(4)$ & -0.003 & $0.003(3)$ & 0.007 (3) \\
\hline C6B & $0.036(4)$ & $0.064(4)$ & 0.039 (4) & $0.000(3)$ & $0.002(3)$ & 0.015 \\
\hline C7B & $0.070(5)$ & $0.052(4)$ & $0.023(4)$ & $0.014(4)$ & $-0.002(3)$ & $0.005(3)$ \\
\hline $\mathrm{C} 8 \mathrm{~B}$ & $0.083(5)$ & 0.031 & $0.034(4)$ & $-0.004(4)$ & $0.001(4)$ & $0.014(3)$ \\
\hline C9B & 0.059 (4) & $0.031(3)$ & $0.034(4)$ & $-0.005(3)$ & $0.000(3)$ & $-0.001(3)$ \\
\hline $\mathrm{C} 10 \mathrm{~B}$ & $0.021(3)$ & 0.021 & $0.067(5)$ & $-0.007(3)$ & 0.005 & $-0.006(3)$ \\
\hline C11B & $0.028(3)$ & $0.034(3)$ & $0.079(5)$ & $-0.002(3)$ & $-0.007(3)$ & $0.001(3)$ \\
\hline C12B & $0.013(3)$ & $0.037(4)$ & $0.130(7)$ & $-0.005(3)$ & $-0.004(4)$ & $0.017(4)$ \\
\hline C13B & $0.028(4)$ & $0.048(4)$ & $0.135(8)$ & 0.009 (3) & $0.017(4)$ & $0.025(5)$ \\
\hline C14B & $0.041(4)$ & $0.043(4)$ & $0.107(6)$ & 0.009 (3) & $0.036(4)$ & 0.018 (4) \\
\hline C15B & $0.042(4)$ & $0.030(3)$ & $0.076(5)$ & $-0.006(3)$ & 0.025 & 0.007 (3) \\
\hline $\mathrm{C} 16 \mathrm{~B}$ & $0.020(3)$ & 0.025 & 0.018 & $-0.008(2)$ & $-0.001(2)$ & $0.003(2)$ \\
\hline C17B & $0.021(3)$ & $0.022(3)$ & $0.037(3)$ & 0.005 (3) & -0.002 & -0.003 \\
\hline $\mathrm{C} 18 \mathrm{~B}$ & $0.027(3)$ & 0.029 (3) & $0.043(4)$ & $0.000(3)$ & -0.008 & $-0.004(3)$ \\
\hline C19B & $0.021(3)$ & $0.042(3)$ & $0.034(4)$ & -0.001 & $-0.004(3)$ & $0.010(3)$ \\
\hline C20B & 0.030 & 0.025 & $0.045(4)$ & $0.008(3)$ & -0.003 & $0.003(3)$ \\
\hline $\mathrm{C} 21 \mathrm{~B}$ & $0.027(3)$ & 0.021 & $0.030(3)$ & $0.004(2)$ & $-0.002(3)$ & $-0.002(3)$ \\
\hline $\mathrm{C} 22 \mathrm{~B}$ & $0.034(3)$ & $0.014(3)$ & $0.024(3)$ & $0.007(2)$ & $0.001(2)$ & $-0.001(2)$ \\
\hline C23B & $0.036(3)$ & $0.032(3)$ & $0.030(3)$ & $0.005(3)$ & 0.008 & $0.003(3)$ \\
\hline C24B & 0.035 & $0.032(3)$ & 0.049 (4) & $-0.004(3)$ & $0.016(3)$ & $0.002(3)$ \\
\hline $\mathrm{C} 25 \mathrm{~B}$ & $0.061(4)$ & $0.032(3)$ & $0.032(4)$ & $0.006(3)$ & 0.019 (3) & $0.006(3)$ \\
\hline $\mathrm{C} 26 \mathrm{~B}$ & $0.066(4)$ & $0.042(4)$ & $0.027(4)$ & $0.013(4)$ & $0.011(3)$ & $0.002(3)$ \\
\hline $\mathrm{C} 27 \mathrm{~B}$ & $0.040(3)$ & $0.018(3)$ & $0.034(4)$ & $0.004(3)$ & $0.000(3)$ & $-0.006(2)$ \\
\hline $\mathrm{C} 28 \mathrm{~B}$ & $0.037(4)$ & $0.068(4)$ & $0.088(5)$ & -0.020 & $-0.026(4)$ & $0.041(4)$ \\
\hline C29B & $0.026(3)$ & $0.026(3)$ & $0.083(5)$ & $-0.004(3)$ & $0.014(3)$ & -0.003 \\
\hline $\mathrm{C} 30 \mathrm{~B}$ & $0.066(5)$ & $0.032(4)$ & $0.108(6)$ & $0.018(4)$ & $-0.037(4)$ & $-0.017(4)$ \\
\hline C31B & $0.091(6)$ & $0.035(4)$ & $0.064(5)$ & $0.003(4)$ & $0.015(4)$ & $0.004(4)$ \\
\hline C32B & $0.086(5)$ & $0.078(5)$ & $0.030(4)$ & $0.036(5)$ & $0.002(4)$ & $0.023(4)$ \\
\hline C33B & $0.048(4)$ & $0.014(3)$ & 0.017 (3) & $0.001(3)$ & $0.005(3)$ & $0.005(2)$ \\
\hline C34B & $0.027(3)$ & $0.021(3)$ & $0.048(4)$ & -0.010 & $-0.007(3)$ & $0.015(3)$ \\
\hline C35B & $0.040(3)$ & $0.024(3)$ & $0.028(3)$ & -0.010 & $0.006(3)$ & $0.001(3)$ \\
\hline $\mathrm{N} 2$ & $0.091(5)$ & $0.217(7)$ & $0.172(7)$ & $0.084(6)$ & $-0.062(5)$ & $-0.147(6)$ \\
\hline
\end{tabular}




\begin{tabular}{lllllll} 
N2B & $0.067(4)$ & $0.124(6)$ & $0.086(6)$ & $0.049(5)$ & $0.001(4)$ & $-0.017(5)$ \\
C36 & $0.071(6)$ & $0.126(7)$ & $0.089(6)$ & $-0.026(6)$ & $0.003(5)$ & $-0.056(5)$ \\
C37 & $0.092(6)$ & $0.068(4)$ & $0.082(6)$ & $0.036(5)$ & $-0.018(5)$ & $-0.057(4)$ \\
C36B & $0.068(6)$ & $0.141(9)$ & $0.087(7)$ & $-0.009(6)$ & $-0.018(5)$ & $0.007(7)$ \\
C37B & $0.069(5)$ & $0.081(5)$ & $0.053(5)$ & $0.035(5)$ & $0.018(4)$ & $0.010(4)$ \\
\hline
\end{tabular}

Geometric parameters $\left(\AA,{ }^{o}\right)$

\begin{tabular}{|c|c|c|c|}
\hline $\mathrm{Ni}-\mathrm{P} 1$ & $2.2012(14)$ & Ni1B-S1B & $2.2495(16)$ \\
\hline $\mathrm{Ni}-\mathrm{P} 2$ & $2.2060(15)$ & Ni1B-S3B & $2.2536(14)$ \\
\hline $\mathrm{Ni}-\mathrm{S} 3$ & $2.2517(13)$ & $\mathrm{Ni1B}-\mathrm{Cl1B}$ & $2.5971(16)$ \\
\hline $\mathrm{Ni}-\mathrm{S} 1$ & $2.2574(15)$ & $\mathrm{Fe} 2 \mathrm{~B}-\mathrm{C} 35 \mathrm{~B}$ & $1.760(6)$ \\
\hline $\mathrm{Ni}-\mathrm{Cl}$ & $2.5787(14)$ & $\mathrm{Fe} 2 \mathrm{~B}-\mathrm{C} 34 \mathrm{~B}$ & $1.912(7)$ \\
\hline $\mathrm{Fe}-\mathrm{C} 35$ & $1.759(6)$ & $\mathrm{Fe} 2 \mathrm{~B}-\mathrm{N} 1 \mathrm{~B}$ & $2.065(5)$ \\
\hline $\mathrm{Fe}-\mathrm{C} 34$ & $1.829(6)$ & $\mathrm{Fe} 2 \mathrm{~B}-\mathrm{S} 2 \mathrm{~B}$ & $2.2839(19)$ \\
\hline $\mathrm{Fe}-\mathrm{N}$ & $2.048(4)$ & $\mathrm{Fe} 2 \mathrm{~B}-\mathrm{S} 1 \mathrm{~B}$ & $2.2909(16)$ \\
\hline $\mathrm{Fe}-\mathrm{S} 1$ & $2.2865(15)$ & $\mathrm{Fe} 2 \mathrm{~B}-\mathrm{S} 3 \mathrm{~B}$ & $2.3029(15)$ \\
\hline $\mathrm{Fe}-\mathrm{S} 2$ & $2.2978(16)$ & $\mathrm{S} 1 \mathrm{~B}-\mathrm{C} 29 \mathrm{~B}$ & $1.821(7)$ \\
\hline $\mathrm{Fe}-\mathrm{S} 3$ & $2.3159(15)$ & $\mathrm{S} 2 \mathrm{~B}-\mathrm{C} 31 \mathrm{~B}$ & $1.844(8)$ \\
\hline $\mathrm{S} 1-\mathrm{C} 29$ & $1.838(6)$ & $\mathrm{S} 3 \mathrm{~B}-\mathrm{C} 33 \mathrm{~B}$ & $1.803(5)$ \\
\hline $\mathrm{S} 2-\mathrm{C} 31$ & $1.831(6)$ & $\mathrm{P} 1 \mathrm{~B}-\mathrm{C} 4 \mathrm{~B}$ & $1.810(6)$ \\
\hline $\mathrm{S} 3-\mathrm{C} 33$ & $1.811(6)$ & $\mathrm{P} 1 \mathrm{~B}-\mathrm{C} 1 \mathrm{~B}$ & $1.812(5)$ \\
\hline $\mathrm{P} 1-\mathrm{C} 4$ & $1.825(5)$ & $\mathrm{P} 1 \mathrm{~B}-\mathrm{C} 10 \mathrm{~B}$ & $1.833(6)$ \\
\hline $\mathrm{P} 1-\mathrm{C} 10$ & $1.839(5)$ & $\mathrm{P} 2 \mathrm{~B}-\mathrm{C} 3 \mathrm{~B}$ & $1.818(5)$ \\
\hline $\mathrm{P} 1-\mathrm{C} 1$ & $1.841(5)$ & $\mathrm{P} 2 \mathrm{~B}-\mathrm{C} 16 \mathrm{~B}$ & $1.830(5)$ \\
\hline $\mathrm{P} 2-\mathrm{C} 3$ & $1.807(5)$ & $\mathrm{P} 2 \mathrm{~B}-\mathrm{C} 22 \mathrm{~B}$ & $1.833(5)$ \\
\hline $\mathrm{P} 2-\mathrm{C} 22$ & $1.825(5)$ & $\mathrm{O} 1 \mathrm{~B}-\mathrm{C} 34 \mathrm{~B}$ & $0.861(8)$ \\
\hline $\mathrm{P} 2-\mathrm{C} 16$ & $1.834(5)$ & $\mathrm{O} 2 \mathrm{~B}-\mathrm{C} 35 \mathrm{~B}$ & $1.137(7)$ \\
\hline $\mathrm{O} 1-\mathrm{C} 34$ & $1.065(7)$ & $\mathrm{N} 1 \mathrm{~B}-\mathrm{C} 30 \mathrm{~B}$ & $1.471(8)$ \\
\hline $\mathrm{O} 2-\mathrm{C} 35$ & $1.151(7)$ & $\mathrm{N} 1 \mathrm{~B}-\mathrm{C} 28 \mathrm{~B}$ & $1.488(8)$ \\
\hline $\mathrm{N}-\mathrm{C} 30$ & $1.499(6)$ & $\mathrm{N} 1 \mathrm{~B}-\mathrm{C} 32 \mathrm{~B}$ & $1.523(8)$ \\
\hline $\mathrm{N}-\mathrm{C} 32$ & $1.508(7)$ & $\mathrm{C} 1 \mathrm{~B}-\mathrm{C} 2 \mathrm{~B}$ & $1.529(8)$ \\
\hline $\mathrm{N}-\mathrm{C} 28$ & $1.527(7)$ & $\mathrm{C} 1 \mathrm{~B}-\mathrm{H} 1 \mathrm{~B} 1$ & 0.99 \\
\hline $\mathrm{C} 1-\mathrm{C} 2$ & $1.505(7)$ & $\mathrm{C} 1 \mathrm{~B}-\mathrm{H} 1 \mathrm{~B} 2$ & 0.99 \\
\hline $\mathrm{C} 1-\mathrm{H} 1 \mathrm{~A}$ & 0.99 & $\mathrm{C} 2 \mathrm{~B}-\mathrm{C} 3 \mathrm{~B}$ & $1.549(7)$ \\
\hline $\mathrm{C} 1-\mathrm{H} 1 \mathrm{~B}$ & 0.99 & $\mathrm{C} 2 \mathrm{~B}-\mathrm{H} 2 \mathrm{~B} 1$ & 0.99 \\
\hline $\mathrm{C} 2-\mathrm{C} 3$ & $1.533(7)$ & $\mathrm{C} 2 \mathrm{~B}-\mathrm{H} 2 \mathrm{~B} 2$ & 0.99 \\
\hline $\mathrm{C} 2-\mathrm{H} 2 \mathrm{~A}$ & 0.99 & $\mathrm{C} 3 \mathrm{~B}-\mathrm{H} 3 \mathrm{~B} 1$ & 0.99 \\
\hline $\mathrm{C} 2-\mathrm{H} 2 \mathrm{~B}$ & 0.99 & $\mathrm{C} 3 \mathrm{~B}-\mathrm{H} 3 \mathrm{~B} 2$ & 0.99 \\
\hline $\mathrm{C} 3-\mathrm{H} 3 \mathrm{~A}$ & 0.99 & $\mathrm{C} 4 \mathrm{~B}-\mathrm{C} 5 \mathrm{~B}$ & $1.367(8)$ \\
\hline $\mathrm{C} 3-\mathrm{H} 3 \mathrm{~B}$ & 0.99 & $\mathrm{C} 4 \mathrm{~B}-\mathrm{C} 9 \mathrm{~B}$ & $1.400(8)$ \\
\hline $\mathrm{C} 4-\mathrm{C} 5$ & $1.384(7)$ & $\mathrm{C} 5 \mathrm{~B}-\mathrm{C} 6 \mathrm{~B}$ & $1.390(8)$ \\
\hline $\mathrm{C} 4-\mathrm{C} 9$ & $1.394(8)$ & $\mathrm{C} 5 \mathrm{~B}-\mathrm{H} 5 \mathrm{~B}$ & 0.95 \\
\hline $\mathrm{C} 5-\mathrm{C} 6$ & $1.394(8)$ & $\mathrm{C} 6 \mathrm{~B}-\mathrm{C} 7 \mathrm{~B}$ & $1.373(9)$ \\
\hline $\mathrm{C} 5-\mathrm{H} 5$ & 0.95 & $\mathrm{C} 6 \mathrm{~B}-\mathrm{H} 6 \mathrm{~B}$ & 0.95 \\
\hline $\mathrm{C} 6-\mathrm{C} 7$ & $1.409(9)$ & $\mathrm{C} 7 \mathrm{~B}-\mathrm{C} 8 \mathrm{~B}$ & $1.387(10)$ \\
\hline C6- 66 & 0.95 & $\mathrm{C} 7 \mathrm{~B}-\mathrm{H} 7 \mathrm{~B}$ & 0.95 \\
\hline $\mathrm{C} 7-\mathrm{C} 8$ & $1.379(9)$ & $\mathrm{C} 8 \mathrm{~B}-\mathrm{C} 9 \mathrm{~B}$ & $1.358(9)$ \\
\hline
\end{tabular}




\begin{tabular}{|c|c|c|c|}
\hline $\mathrm{C} 7-\mathrm{H} 7$ & 0.95 & $\mathrm{C} 8 \mathrm{~B}-\mathrm{H} 8 \mathrm{~B}$ & 0.95 \\
\hline $\mathrm{C} 8-\mathrm{C} 9$ & $1.365(8)$ & $\mathrm{C} 9 \mathrm{~B}-\mathrm{H} 9 \mathrm{~B}$ & 0.95 \\
\hline $\mathrm{C} 8-\mathrm{H} 8$ & 0.95 & $\mathrm{C} 10 \mathrm{~B}-\mathrm{C} 15 \mathrm{~B}$ & $1.387(9)$ \\
\hline C9-H9 & 0.95 & $\mathrm{C} 10 \mathrm{~B}-\mathrm{C} 11 \mathrm{~B}$ & $1.415(9)$ \\
\hline $\mathrm{C} 10-\mathrm{C} 15$ & $1.382(7)$ & $\mathrm{C} 11 \mathrm{~B}-\mathrm{C} 12 \mathrm{~B}$ & $1.384(9)$ \\
\hline $\mathrm{C} 10-\mathrm{C} 11$ & $1.392(8)$ & C11B-H11B & 0.95 \\
\hline $\mathrm{C} 11-\mathrm{C} 12$ & $1.391(8)$ & $\mathrm{C} 12 \mathrm{~B}-\mathrm{C} 13 \mathrm{~B}$ & $1.366(12)$ \\
\hline C11-H11 & 0.95 & $\mathrm{C} 12 \mathrm{~B}-\mathrm{H} 12 \mathrm{~B}$ & 0.95 \\
\hline $\mathrm{C} 12-\mathrm{C} 13$ & $1.370(9)$ & $\mathrm{C} 13 \mathrm{~B}-\mathrm{C} 14 \mathrm{~B}$ & $1.371(11)$ \\
\hline $\mathrm{C} 12-\mathrm{H} 12$ & 0.95 & $\mathrm{C} 13 \mathrm{~B}-\mathrm{H} 13 \mathrm{~B}$ & 0.95 \\
\hline $\mathrm{C} 13-\mathrm{C} 14$ & $1.368(8)$ & $\mathrm{C} 14 \mathrm{~B}-\mathrm{C} 15 \mathrm{~B}$ & $1.431(9)$ \\
\hline C13-H13 & 0.95 & $\mathrm{C} 14 \mathrm{~B}-\mathrm{H} 14 \mathrm{~B}$ & 0.95 \\
\hline $\mathrm{C} 14-\mathrm{C} 15$ & $1.396(8)$ & $\mathrm{C} 15 \mathrm{~B}-\mathrm{H} 15 \mathrm{~B}$ & 0.95 \\
\hline C14-H14 & 0.95 & $\mathrm{C} 16 \mathrm{~B}-\mathrm{C} 21 \mathrm{~B}$ & $1.395(7)$ \\
\hline $\mathrm{C} 15-\mathrm{H} 15$ & 0.95 & $\mathrm{C} 16 \mathrm{~B}-\mathrm{C} 17 \mathrm{~B}$ & $1.404(7)$ \\
\hline $\mathrm{C} 16-\mathrm{C} 17$ & $1.374(7)$ & $\mathrm{C} 17 \mathrm{~B}-\mathrm{C} 18 \mathrm{~B}$ & $1.343(8)$ \\
\hline $\mathrm{C} 16-\mathrm{C} 21$ & $1.382(7)$ & $\mathrm{C} 17 \mathrm{~B}-\mathrm{H} 17 \mathrm{~B}$ & 0.95 \\
\hline $\mathrm{C} 17-\mathrm{C} 18$ & $1.391(8)$ & $\mathrm{C} 18 \mathrm{~B}-\mathrm{C} 19 \mathrm{~B}$ & $1.358(8)$ \\
\hline $\mathrm{C} 17-\mathrm{H} 17$ & 0.95 & $\mathrm{C} 18 \mathrm{~B}-\mathrm{H} 18 \mathrm{~B}$ & 0.95 \\
\hline $\mathrm{C} 18-\mathrm{C} 19$ & $1.373(8)$ & $\mathrm{C} 19 \mathrm{~B}-\mathrm{C} 20 \mathrm{~B}$ & $1.391(8)$ \\
\hline $\mathrm{C} 18-\mathrm{H} 18$ & 0.95 & C19B-H19B & 0.95 \\
\hline $\mathrm{C} 19-\mathrm{C} 20$ & $1.380(8)$ & $\mathrm{C} 20 \mathrm{~B}-\mathrm{C} 21 \mathrm{~B}$ & $1.386(8)$ \\
\hline C19-H19 & 0.95 & $\mathrm{C} 20 \mathrm{~B}-\mathrm{H} 20 \mathrm{~B}$ & 0.95 \\
\hline $\mathrm{C} 20-\mathrm{C} 21$ & $1.397(8)$ & $\mathrm{C} 21 \mathrm{~B}-\mathrm{H} 21 \mathrm{~B}$ & 0.95 \\
\hline $\mathrm{C} 20-\mathrm{H} 20$ & 0.95 & $\mathrm{C} 22 \mathrm{~B}-\mathrm{C} 23 \mathrm{~B}$ & $1.384(8)$ \\
\hline $\mathrm{C} 21-\mathrm{H} 21$ & 0.95 & $\mathrm{C} 22 \mathrm{~B}-\mathrm{C} 27 \mathrm{~B}$ & $1.406(8)$ \\
\hline $\mathrm{C} 22-\mathrm{C} 23$ & $1.380(8)$ & $\mathrm{C} 23 \mathrm{~B}-\mathrm{C} 24 \mathrm{~B}$ & $1.391(8)$ \\
\hline $\mathrm{C} 22-\mathrm{C} 27$ & $1.384(8)$ & $\mathrm{C} 23 \mathrm{~B}-\mathrm{H} 23 \mathrm{~B}$ & 0.95 \\
\hline $\mathrm{C} 23-\mathrm{C} 24$ & $1.395(8)$ & $\mathrm{C} 24 \mathrm{~B}-\mathrm{C} 25 \mathrm{~B}$ & $1.355(9)$ \\
\hline $\mathrm{C} 23-\mathrm{H} 23$ & 0.95 & $\mathrm{C} 24 \mathrm{~B}-\mathrm{H} 24 \mathrm{~B}$ & 0.95 \\
\hline $\mathrm{C} 24-\mathrm{C} 25$ & $1.373(9)$ & $\mathrm{C} 25 \mathrm{~B}-\mathrm{C} 26 \mathrm{~B}$ & $1.399(9)$ \\
\hline $\mathrm{C} 24-\mathrm{H} 24$ & 0.95 & $\mathrm{C} 25 \mathrm{~B}-\mathrm{H} 25 \mathrm{~B}$ & 0.95 \\
\hline $\mathrm{C} 25-\mathrm{C} 26$ & $1.366(9)$ & $\mathrm{C} 26 \mathrm{~B}-\mathrm{C} 27 \mathrm{~B}$ & $1.369(9)$ \\
\hline $\mathrm{C} 25-\mathrm{H} 25$ & 0.95 & $\mathrm{C} 26 \mathrm{~B}-\mathrm{H} 26 \mathrm{~B}$ & 0.95 \\
\hline $\mathrm{C} 26-\mathrm{C} 27$ & $1.400(8)$ & $\mathrm{C} 27 \mathrm{~B}-\mathrm{H} 27 \mathrm{~B}$ & 0.95 \\
\hline $\mathrm{C} 26-\mathrm{H} 26$ & 0.95 & $\mathrm{C} 28 \mathrm{~B}-\mathrm{C} 29 \mathrm{~B}$ & $1.445(9)$ \\
\hline $\mathrm{C} 27-\mathrm{H} 27$ & 0.95 & $\mathrm{C} 28 \mathrm{~B}-\mathrm{H} 28 \mathrm{C}$ & 0.99 \\
\hline $\mathrm{C} 28-\mathrm{C} 29$ & $1.514(8)$ & $\mathrm{C} 28 \mathrm{~B}-\mathrm{H} 28 \mathrm{D}$ & 0.99 \\
\hline $\mathrm{C} 28-\mathrm{H} 28 \mathrm{~A}$ & 0.99 & $\mathrm{C} 29 \mathrm{~B}-\mathrm{H} 29 \mathrm{C}$ & 0.99 \\
\hline $\mathrm{C} 28-\mathrm{H} 28 \mathrm{~B}$ & 0.99 & $\mathrm{C} 29 \mathrm{~B}-\mathrm{H} 29 \mathrm{D}$ & 0.99 \\
\hline $\mathrm{C} 29-\mathrm{H} 29 \mathrm{~A}$ & 0.99 & $\mathrm{C} 30 \mathrm{~B}-\mathrm{C} 31 \mathrm{~B}$ & $1.591(11)$ \\
\hline $\mathrm{C} 29-\mathrm{H} 29 \mathrm{~B}$ & 0.99 & $\mathrm{C} 30 \mathrm{~B}-\mathrm{H} 30 \mathrm{C}$ & 0.99 \\
\hline C30-C31 & $1.525(8)$ & $\mathrm{C} 30 \mathrm{~B}-\mathrm{H} 30 \mathrm{D}$ & 0.99 \\
\hline $\mathrm{C} 30-\mathrm{H} 30 \mathrm{~A}$ & 0.99 & $\mathrm{C} 31 \mathrm{~B}-\mathrm{H} 31 \mathrm{C}$ & 0.99 \\
\hline $\mathrm{C} 30-\mathrm{H} 30 \mathrm{~B}$ & 0.99 & $\mathrm{C} 31 \mathrm{~B}-\mathrm{H} 31 \mathrm{D}$ & 0.99 \\
\hline $\mathrm{C} 31-\mathrm{H} 31 \mathrm{~A}$ & 0.99 & $\mathrm{C} 32 \mathrm{~B}-\mathrm{C} 33 \mathrm{~B}$ & $1.464(9)$ \\
\hline $\mathrm{C} 31-\mathrm{H} 31 \mathrm{~B}$ & 0.99 & $\mathrm{C} 32 \mathrm{~B}-\mathrm{H} 32 \mathrm{C}$ & 0.99 \\
\hline C32-C33 & $1.514(8)$ & $\mathrm{C} 32 \mathrm{~B}-\mathrm{H} 32 \mathrm{D}$ & 0.99 \\
\hline
\end{tabular}




\begin{tabular}{|c|c|c|c|}
\hline $\mathrm{C} 32-\mathrm{H} 32 \mathrm{~A}$ & 0.99 & $\mathrm{C} 33 \mathrm{~B}-\mathrm{H} 33 \mathrm{C}$ & 0.99 \\
\hline C $32-\mathrm{H} 32 \mathrm{~B}$ & 0.99 & $\mathrm{C} 33 \mathrm{~B}-\mathrm{H} 33 \mathrm{D}$ & 0.99 \\
\hline C $33-\mathrm{H} 33 \mathrm{~A}$ & 0.99 & $\mathrm{~N} 2-\mathrm{C} 37$ & $1.160(11)$ \\
\hline С $33-\mathrm{H} 33 \mathrm{~B}$ & 0.99 & $\mathrm{~N} 2 \mathrm{~B}-\mathrm{C} 37 \mathrm{~B}$ & $1.141(11)$ \\
\hline Ni1B-P1B & $2.2018(15)$ & $\mathrm{C} 36-\mathrm{C} 37$ & $1.462(13)$ \\
\hline Ni1B-P2B & $2.2046(15)$ & $\mathrm{C} 36 \mathrm{~B}-\mathrm{C} 37 \mathrm{~B}$ & $1.439(12)$ \\
\hline $\mathrm{P} 1-\mathrm{Ni}-\mathrm{P} 2$ & $92.88(5)$ & $\mathrm{P} 1 \mathrm{~B}-\mathrm{Ni1B}-\mathrm{S} 1 \mathrm{~B}$ & $87.92(6)$ \\
\hline $\mathrm{P} 1-\mathrm{Ni}-\mathrm{S} 3$ & $165.43(6)$ & $\mathrm{P} 2 \mathrm{~B}-\mathrm{Ni} 1 \mathrm{~B}-\mathrm{S} 1 \mathrm{~B}$ & $161.55(6)$ \\
\hline $\mathrm{P} 2-\mathrm{Ni}-\mathrm{S} 3$ & $88.90(5)$ & $\mathrm{P} 1 \mathrm{~B}-\mathrm{Ni1B}-\mathrm{S} 3 \mathrm{~B}$ & $164.85(6)$ \\
\hline $\mathrm{P} 1-\mathrm{Ni}-\mathrm{S} 1$ & $87.87(5)$ & $\mathrm{P} 2 \mathrm{~B}-\mathrm{Ni1B}-\mathrm{S} 3 \mathrm{~B}$ & $88.78(5)$ \\
\hline $\mathrm{P} 2-\mathrm{Ni}-\mathrm{S} 1$ & $160.38(6)$ & $\mathrm{S} 1 \mathrm{~B}-\mathrm{Ni1B}-\mathrm{S} 3 \mathrm{~B}$ & $85.85(5)$ \\
\hline $\mathrm{S} 3-\mathrm{Ni}-\mathrm{S} 1$ & $85.67(5)$ & $\mathrm{P} 1 \mathrm{~B}-\mathrm{Ni1B}-\mathrm{C} 11 \mathrm{~B}$ & $88.79(5)$ \\
\hline $\mathrm{P} 1-\mathrm{Ni}-\mathrm{Cl}$ & $88.51(5)$ & $\mathrm{P} 2 \mathrm{~B}-\mathrm{Ni1B}-\mathrm{C} 11 \mathrm{~B}$ & $92.29(5)$ \\
\hline $\mathrm{P} 2-\mathrm{Ni}-\mathrm{Cl}$ & $92.94(5)$ & $\mathrm{S} 1 \mathrm{~B}-\mathrm{Ni1B}-\mathrm{C} 11 \mathrm{~B}$ & $106.16(6)$ \\
\hline $\mathrm{S} 3-\mathrm{Ni}-\mathrm{Cl}$ & $105.85(5)$ & $\mathrm{S} 3 \mathrm{~B}-\mathrm{Ni1B}-\mathrm{C} 11 \mathrm{~B}$ & $106.21(5)$ \\
\hline $\mathrm{S} 1-\mathrm{Ni}-\mathrm{Cl}$ & $106.68(5)$ & $\mathrm{C} 35 \mathrm{~B}-\mathrm{Fe} 2 \mathrm{~B}-\mathrm{C} 34 \mathrm{~B}$ & $90.1(3)$ \\
\hline $\mathrm{C} 35-\mathrm{Fe}-\mathrm{C} 34$ & $91.6(2)$ & $\mathrm{C} 35 \mathrm{~B}-\mathrm{Fe} 2 \mathrm{~B}-\mathrm{N} 1 \mathrm{~B}$ & $177.9(2)$ \\
\hline $\mathrm{C} 35-\mathrm{Fe}-\mathrm{N}$ & $175.2(2)$ & $\mathrm{C} 34 \mathrm{~B}-\mathrm{Fe} 2 \mathrm{~B}-\mathrm{N} 1 \mathrm{~B}$ & $91.8(2)$ \\
\hline $\mathrm{C} 34-\mathrm{Fe}-\mathrm{N}$ & $92.5(2)$ & $\mathrm{C} 35 \mathrm{~B}-\mathrm{Fe} 2 \mathrm{~B}-\mathrm{S} 2 \mathrm{~B}$ & $94.41(19)$ \\
\hline $\mathrm{C} 35-\mathrm{Fe}-\mathrm{S} 1$ & $95.72(19)$ & $\mathrm{C} 34 \mathrm{~B}-\mathrm{Fe} 2 \mathrm{~B}-\mathrm{S} 2 \mathrm{~B}$ & $87.86(18)$ \\
\hline $\mathrm{C} 34-\mathrm{Fe}-\mathrm{S} 1$ & $96.10(18)$ & $\mathrm{N} 1 \mathrm{~B}-\mathrm{Fe} 2 \mathrm{~B}-\mathrm{S} 2 \mathrm{~B}$ & $86.57(13)$ \\
\hline $\mathrm{N}-\mathrm{Fe}-\mathrm{S} 1$ & $86.26(12)$ & $\mathrm{C} 35 \mathrm{~B}-\mathrm{Fe} 2 \mathrm{~B}-\mathrm{S} 1 \mathrm{~B}$ & $92.44(18)$ \\
\hline $\mathrm{C} 35-\mathrm{Fe}-\mathrm{S} 2$ & $90.91(19)$ & $\mathrm{C} 34 \mathrm{~B}-\mathrm{Fe} 2 \mathrm{~B}-\mathrm{S} 1 \mathrm{~B}$ & $95.97(18)$ \\
\hline $\mathrm{C} 34-\mathrm{Fe}-\mathrm{S} 2$ & $87.63(18)$ & $\mathrm{N} 1 \mathrm{~B}-\mathrm{Fe} 2 \mathrm{~B}-\mathrm{S} 1 \mathrm{~B}$ & $86.47(13)$ \\
\hline $\mathrm{N}-\mathrm{Fe}-\mathrm{S} 2$ & $86.83(12)$ & $\mathrm{S} 2 \mathrm{~B}-\mathrm{Fe} 2 \mathrm{~B}-\mathrm{S} 1 \mathrm{~B}$ & $172.15(7)$ \\
\hline $\mathrm{S} 1-\mathrm{Fe}-\mathrm{S} 2$ & $172.29(7)$ & $\mathrm{C} 35 \mathrm{~B}-\mathrm{Fe} 2 \mathrm{~B}-\mathrm{S} 3 \mathrm{~B}$ & $90.78(19)$ \\
\hline $\mathrm{C} 35-\mathrm{Fe}-\mathrm{S} 3$ & $88.34(18)$ & $\mathrm{C} 34 \mathrm{~B}-\mathrm{Fe} 2 \mathrm{~B}-\mathrm{S} 3 \mathrm{~B}$ & $179.1(2)$ \\
\hline $\mathrm{C} 34-\mathrm{Fe}-\mathrm{S} 3$ & $179.60(19)$ & $\mathrm{N} 1 \mathrm{~B}-\mathrm{Fe} 2 \mathrm{~B}-\mathrm{S} 3 \mathrm{~B}$ & $87.29(13)$ \\
\hline $\mathrm{N}-\mathrm{Fe}-\mathrm{S} 3$ & $87.58(12)$ & $\mathrm{S} 2 \mathrm{~B}-\mathrm{Fe} 2 \mathrm{~B}-\mathrm{S} 3 \mathrm{~B}$ & $92.30(6)$ \\
\hline $\mathrm{S} 1-\mathrm{Fe}-\mathrm{S} 3$ & $83.53(5)$ & $\mathrm{S} 1 \mathrm{~B}-\mathrm{Fe} 2 \mathrm{~B}-\mathrm{S} 3 \mathrm{~B}$ & $83.76(5)$ \\
\hline $\mathrm{S} 2-\mathrm{Fe}-\mathrm{S} 3$ & $92.76(6)$ & $\mathrm{C} 29 \mathrm{~B}-\mathrm{S} 1 \mathrm{~B}-\mathrm{Ni1B}$ & $106.3(2)$ \\
\hline $\mathrm{C} 29-\mathrm{S} 1-\mathrm{Ni}$ & $105.5(2)$ & $\mathrm{C} 29 \mathrm{~B}-\mathrm{S} 1 \mathrm{~B}-\mathrm{Fe} 2 \mathrm{~B}$ & $99.02(19)$ \\
\hline $\mathrm{C} 29-\mathrm{S} 1-\mathrm{Fe}$ & $99.79(18)$ & $\mathrm{Ni1B}-\mathrm{S} 1 \mathrm{~B}-\mathrm{Fe} 2 \mathrm{~B}$ & $95.18(6)$ \\
\hline $\mathrm{Ni}-\mathrm{S} 1-\mathrm{Fe}$ & $95.51(6)$ & $\mathrm{C} 31 \mathrm{~B}-\mathrm{S} 2 \mathrm{~B}-\mathrm{Fe} 2 \mathrm{~B}$ & $98.7(2)$ \\
\hline $\mathrm{C} 31-\mathrm{S} 2-\mathrm{Fe}$ & $98.65(18)$ & $\mathrm{C} 33 \mathrm{~B}-\mathrm{S} 3 \mathrm{~B}-\mathrm{Ni1B}$ & $108.53(19)$ \\
\hline $\mathrm{C} 33-\mathrm{S} 3-\mathrm{Ni}$ & $109.84(18)$ & $\mathrm{C} 33 \mathrm{~B}-\mathrm{S} 3 \mathrm{~B}-\mathrm{Fe} 2 \mathrm{~B}$ & $97.50(18)$ \\
\hline $\mathrm{C} 33-\mathrm{S} 3-\mathrm{Fe}$ & $96.70(17)$ & $\mathrm{Ni} 1 \mathrm{~B}-\mathrm{S} 3 \mathrm{~B}-\mathrm{Fe} 2 \mathrm{~B}$ & $94.74(5)$ \\
\hline $\mathrm{Ni}-\mathrm{S} 3-\mathrm{Fe}$ & $94.85(5)$ & $\mathrm{C} 4 \mathrm{~B}-\mathrm{P} 1 \mathrm{~B}-\mathrm{C} 1 \mathrm{~B}$ & $103.1(2)$ \\
\hline $\mathrm{C} 4-\mathrm{P} 1-\mathrm{C} 10$ & $104.3(2)$ & $\mathrm{C} 4 \mathrm{~B}-\mathrm{P} 1 \mathrm{~B}-\mathrm{C} 10 \mathrm{~B}$ & $103.2(3)$ \\
\hline $\mathrm{C} 4-\mathrm{P} 1-\mathrm{C} 1$ & $103.2(2)$ & $\mathrm{C} 1 \mathrm{~B}-\mathrm{P} 1 \mathrm{~B}-\mathrm{C} 10 \mathrm{~B}$ & $103.0(3)$ \\
\hline $\mathrm{C} 10-\mathrm{P} 1-\mathrm{C} 1$ & $101.9(2)$ & $\mathrm{C} 4 \mathrm{~B}-\mathrm{P} 1 \mathrm{~B}-\mathrm{Ni1B}$ & $114.42(18)$ \\
\hline $\mathrm{C} 4-\mathrm{P} 1-\mathrm{Ni}$ & $113.45(17)$ & $\mathrm{C} 1 \mathrm{~B}-\mathrm{P} 1 \mathrm{~B}-\mathrm{Ni1B}$ & $115.71(18)$ \\
\hline $\mathrm{C} 10-\mathrm{P} 1-\mathrm{Ni}$ & $116.11(16)$ & $\mathrm{C} 10 \mathrm{~B}-\mathrm{P} 1 \mathrm{~B}-\mathrm{Ni1B}$ & $115.63(19)$ \\
\hline $\mathrm{C} 1-\mathrm{P} 1-\mathrm{Ni}$ & $116.24(18)$ & $\mathrm{C} 3 \mathrm{~B}-\mathrm{P} 2 \mathrm{~B}-\mathrm{C} 16 \mathrm{~B}$ & $105.9(2)$ \\
\hline $\mathrm{C} 3-\mathrm{P} 2-\mathrm{C} 22$ & $101.7(2)$ & $\mathrm{C} 3 \mathrm{~B}-\mathrm{P} 2 \mathrm{~B}-\mathrm{C} 22 \mathrm{~B}$ & $102.3(2)$ \\
\hline $\mathrm{C} 3-\mathrm{P} 2-\mathrm{C} 16$ & $105.9(2)$ & $\mathrm{C} 16 \mathrm{~B}-\mathrm{P} 2 \mathrm{~B}-\mathrm{C} 22 \mathrm{~B}$ & $103.9(2)$ \\
\hline $\mathrm{C} 22-\mathrm{P} 2-\mathrm{C} 16$ & $104.3(2)$ & $\mathrm{C} 3 \mathrm{~B}-\mathrm{P} 2 \mathrm{~B}-\mathrm{Ni1B}$ & $116.50(18)$ \\
\hline
\end{tabular}




\begin{tabular}{|c|c|c|c|}
\hline $\mathrm{C} 3-\mathrm{P} 2-\mathrm{Ni}$ & $115.61(18)$ & $\mathrm{C} 16 \mathrm{~B}-\mathrm{P} 2 \mathrm{~B}-\mathrm{Ni1B}$ & $111.67(17)$ \\
\hline $\mathrm{C} 22-\mathrm{P} 2-\mathrm{Ni}$ & $115.54(17)$ & $\mathrm{C} 22 \mathrm{~B}-\mathrm{P} 2 \mathrm{~B}-\mathrm{Ni1B}$ & $115.26(17)$ \\
\hline $\mathrm{C} 16-\mathrm{P} 2-\mathrm{Ni}$ & $112.53(17)$ & $\mathrm{C} 30 \mathrm{~B}-\mathrm{N} 1 \mathrm{~B}-\mathrm{C} 28 \mathrm{~B}$ & $108.2(5)$ \\
\hline $\mathrm{C} 30-\mathrm{N}-\mathrm{C} 32$ & $110.8(4)$ & $\mathrm{C} 30 \mathrm{~B}-\mathrm{N} 1 \mathrm{~B}-\mathrm{C} 32 \mathrm{~B}$ & $112.5(5)$ \\
\hline $\mathrm{C} 30-\mathrm{N}-\mathrm{C} 28$ & $106.6(4)$ & $\mathrm{C} 28 \mathrm{~B}-\mathrm{N} 1 \mathrm{~B}-\mathrm{C} 32 \mathrm{~B}$ & $103.2(5)$ \\
\hline $\mathrm{C} 32-\mathrm{N}-\mathrm{C} 28$ & $105.9(4)$ & $\mathrm{C} 30 \mathrm{~B}-\mathrm{N} 1 \mathrm{~B}-\mathrm{Fe} 2 \mathrm{~B}$ & $109.8(4)$ \\
\hline $\mathrm{C} 30-\mathrm{N}-\mathrm{Fe}$ & $109.4(3)$ & $\mathrm{C} 28 \mathrm{~B}-\mathrm{N} 1 \mathrm{~B}-\mathrm{Fe} 2 \mathrm{~B}$ & $109.3(4)$ \\
\hline $\mathrm{C} 32-\mathrm{N}-\mathrm{Fe}$ & $114.3(3)$ & $\mathrm{C} 32 \mathrm{~B}-\mathrm{N} 1 \mathrm{~B}-\mathrm{Fe} 2 \mathrm{~B}$ & $113.5(4)$ \\
\hline $\mathrm{C} 28-\mathrm{N}-\mathrm{Fe}$ & $109.4(3)$ & $\mathrm{C} 2 \mathrm{~B}-\mathrm{C} 1 \mathrm{~B}-\mathrm{P} 1 \mathrm{~B}$ & $114.5(4)$ \\
\hline $\mathrm{C} 2-\mathrm{C} 1-\mathrm{P} 1$ & $114.5(4)$ & $\mathrm{C} 2 \mathrm{~B}-\mathrm{C} 1 \mathrm{~B}-\mathrm{H} 1 \mathrm{~B} 1$ & 108.6 \\
\hline $\mathrm{C} 2-\mathrm{C} 1-\mathrm{H} 1 \mathrm{~A}$ & 108.6 & $\mathrm{P} 1 \mathrm{~B}-\mathrm{C} 1 \mathrm{~B}-\mathrm{H} 1 \mathrm{~B} 1$ & 108.6 \\
\hline $\mathrm{P} 1-\mathrm{C} 1-\mathrm{H} 1 \mathrm{~A}$ & 108.6 & $\mathrm{C} 2 \mathrm{~B}-\mathrm{C} 1 \mathrm{~B}-\mathrm{H} 1 \mathrm{~B} 2$ & 108.6 \\
\hline $\mathrm{C} 2-\mathrm{C} 1-\mathrm{H} 1 \mathrm{~B}$ & 108.6 & $\mathrm{P} 1 \mathrm{~B}-\mathrm{C} 1 \mathrm{~B}-\mathrm{H} 1 \mathrm{~B} 2$ & 108.6 \\
\hline $\mathrm{P} 1-\mathrm{C} 1-\mathrm{H} 1 \mathrm{~B}$ & 108.6 & $\mathrm{H} 1 \mathrm{~B} 1-\mathrm{C} 1 \mathrm{~B}-\mathrm{H} 1 \mathrm{~B} 2$ & 107.6 \\
\hline $\mathrm{H} 1 \mathrm{~A}-\mathrm{C} 1-\mathrm{H} 1 \mathrm{~B}$ & 107.6 & $\mathrm{C} 1 \mathrm{~B}-\mathrm{C} 2 \mathrm{~B}-\mathrm{C} 3 \mathrm{~B}$ & $114.4(4)$ \\
\hline $\mathrm{C} 1-\mathrm{C} 2-\mathrm{C} 3$ & $113.5(4)$ & $\mathrm{C} 1 \mathrm{~B}-\mathrm{C} 2 \mathrm{~B}-\mathrm{H} 2 \mathrm{~B} 1$ & 108.7 \\
\hline $\mathrm{C} 1-\mathrm{C} 2-\mathrm{H} 2 \mathrm{~A}$ & 108.9 & $\mathrm{C} 3 \mathrm{~B}-\mathrm{C} 2 \mathrm{~B}-\mathrm{H} 2 \mathrm{~B} 1$ & 108.7 \\
\hline $\mathrm{C} 3-\mathrm{C} 2-\mathrm{H} 2 \mathrm{~A}$ & 108.9 & $\mathrm{C} 1 \mathrm{~B}-\mathrm{C} 2 \mathrm{~B}-\mathrm{H} 2 \mathrm{~B} 2$ & 108.7 \\
\hline $\mathrm{C} 1-\mathrm{C} 2-\mathrm{H} 2 \mathrm{~B}$ & 108.9 & $\mathrm{C} 3 \mathrm{~B}-\mathrm{C} 2 \mathrm{~B}-\mathrm{H} 2 \mathrm{~B} 2$ & 108.7 \\
\hline $\mathrm{C} 3-\mathrm{C} 2-\mathrm{H} 2 \mathrm{~B}$ & 108.9 & $\mathrm{H} 2 \mathrm{~B} 1-\mathrm{C} 2 \mathrm{~B}-\mathrm{H} 2 \mathrm{~B} 2$ & 107.6 \\
\hline $\mathrm{H} 2 \mathrm{~A}-\mathrm{C} 2-\mathrm{H} 2 \mathrm{~B}$ & 107.7 & $\mathrm{C} 2 \mathrm{~B}-\mathrm{C} 3 \mathrm{~B}-\mathrm{P} 2 \mathrm{~B}$ & $114.7(4)$ \\
\hline $\mathrm{C} 2-\mathrm{C} 3-\mathrm{P} 2$ & $115.9(4)$ & $\mathrm{C} 2 \mathrm{~B}-\mathrm{C} 3 \mathrm{~B}-\mathrm{H} 3 \mathrm{~B} 1$ & 108.6 \\
\hline $\mathrm{C} 2-\mathrm{C} 3-\mathrm{H} 3 \mathrm{~A}$ & 108.3 & $\mathrm{P} 2 \mathrm{~B}-\mathrm{C} 3 \mathrm{~B}-\mathrm{H} 3 \mathrm{~B} 1$ & 108.6 \\
\hline $\mathrm{P} 2-\mathrm{C} 3-\mathrm{H} 3 \mathrm{~A}$ & 108.3 & $\mathrm{C} 2 \mathrm{~B}-\mathrm{C} 3 \mathrm{~B}-\mathrm{H} 3 \mathrm{~B} 2$ & 108.6 \\
\hline $\mathrm{C} 2-\mathrm{C} 3-\mathrm{H} 3 \mathrm{~B}$ & 108.3 & $\mathrm{P} 2 \mathrm{~B}-\mathrm{C} 3 \mathrm{~B}-\mathrm{H} 3 \mathrm{~B} 2$ & 108.6 \\
\hline $\mathrm{P} 2-\mathrm{C} 3-\mathrm{H} 3 \mathrm{~B}$ & 108.3 & $\mathrm{H} 3 \mathrm{~B} 1-\mathrm{C} 3 \mathrm{~B}-\mathrm{H} 3 \mathrm{~B} 2$ & 107.6 \\
\hline $\mathrm{H} 3 \mathrm{~A}-\mathrm{C} 3-\mathrm{H} 3 \mathrm{~B}$ & 107.4 & $\mathrm{C} 5 \mathrm{~B}-\mathrm{C} 4 \mathrm{~B}-\mathrm{C} 9 \mathrm{~B}$ & $116.8(5)$ \\
\hline $\mathrm{C} 5-\mathrm{C} 4-\mathrm{C} 9$ & $118.9(5)$ & $\mathrm{C} 5 \mathrm{~B}-\mathrm{C} 4 \mathrm{~B}-\mathrm{P} 1 \mathrm{~B}$ & $121.2(4)$ \\
\hline $\mathrm{C} 5-\mathrm{C} 4-\mathrm{P} 1$ & $121.0(4)$ & $\mathrm{C} 9 \mathrm{~B}-\mathrm{C} 4 \mathrm{~B}-\mathrm{P} 1 \mathrm{~B}$ & $121.9(4)$ \\
\hline $\mathrm{C} 9-\mathrm{C} 4-\mathrm{P} 1$ & $120.0(4)$ & $\mathrm{C} 4 \mathrm{~B}-\mathrm{C} 5 \mathrm{~B}-\mathrm{C} 6 \mathrm{~B}$ & $122.0(6)$ \\
\hline $\mathrm{C} 4-\mathrm{C} 5-\mathrm{C} 6$ & $120.6(6)$ & $\mathrm{C} 4 \mathrm{~B}-\mathrm{C} 5 \mathrm{~B}-\mathrm{H} 5 \mathrm{~B}$ & 119 \\
\hline $\mathrm{C} 4-\mathrm{C} 5-\mathrm{H} 5$ & 119.7 & $\mathrm{C} 6 \mathrm{~B}-\mathrm{C} 5 \mathrm{~B}-\mathrm{H} 5 \mathrm{~B}$ & 119 \\
\hline $\mathrm{C} 6-\mathrm{C} 5-\mathrm{H} 5$ & 119.7 & $\mathrm{C} 7 \mathrm{~B}-\mathrm{C} 6 \mathrm{~B}-\mathrm{C} 5 \mathrm{~B}$ & $120.2(6)$ \\
\hline $\mathrm{C} 5-\mathrm{C} 6-\mathrm{C} 7$ & $118.5(6)$ & $\mathrm{C} 7 \mathrm{~B}-\mathrm{C} 6 \mathrm{~B}-\mathrm{H} 6 \mathrm{~B}$ & 119.9 \\
\hline $\mathrm{C} 5-\mathrm{C} 6-\mathrm{H} 6$ & 120.7 & $\mathrm{C} 5 \mathrm{~B}-\mathrm{C} 6 \mathrm{~B}-\mathrm{H} 6 \mathrm{~B}$ & 119.9 \\
\hline $\mathrm{C} 7-\mathrm{C} 6-\mathrm{H} 6$ & 120.7 & $\mathrm{C} 6 \mathrm{~B}-\mathrm{C} 7 \mathrm{~B}-\mathrm{C} 8 \mathrm{~B}$ & $118.2(6)$ \\
\hline $\mathrm{C} 8-\mathrm{C} 7-\mathrm{C} 6$ & $120.6(6)$ & $\mathrm{C} 6 \mathrm{~B}-\mathrm{C} 7 \mathrm{~B}-\mathrm{H} 7 \mathrm{~B}$ & 120.9 \\
\hline $\mathrm{C} 8-\mathrm{C} 7-\mathrm{H} 7$ & 119.7 & $\mathrm{C} 8 \mathrm{~B}-\mathrm{C} 7 \mathrm{~B}-\mathrm{H} 7 \mathrm{~B}$ & 120.9 \\
\hline $\mathrm{C} 6-\mathrm{C} 7-\mathrm{H} 7$ & 119.7 & $\mathrm{C} 9 \mathrm{~B}-\mathrm{C} 8 \mathrm{~B}-\mathrm{C} 7 \mathrm{~B}$ & $121.0(6)$ \\
\hline $\mathrm{C} 9-\mathrm{C} 8-\mathrm{C} 7$ & $119.7(6)$ & $\mathrm{C} 9 \mathrm{~B}-\mathrm{C} 8 \mathrm{~B}-\mathrm{H} 8 \mathrm{~B}$ & 119.5 \\
\hline $\mathrm{C} 9-\mathrm{C} 8-\mathrm{H} 8$ & 120.2 & $\mathrm{C} 7 \mathrm{~B}-\mathrm{C} 8 \mathrm{~B}-\mathrm{H} 8 \mathrm{~B}$ & 119.5 \\
\hline $\mathrm{C} 7-\mathrm{C} 8-\mathrm{H} 8$ & 120.2 & $\mathrm{C} 8 \mathrm{~B}-\mathrm{C} 9 \mathrm{~B}-\mathrm{C} 4 \mathrm{~B}$ & $121.6(6)$ \\
\hline $\mathrm{C} 8-\mathrm{C} 9-\mathrm{C} 4$ & $121.4(6)$ & $\mathrm{C} 8 \mathrm{~B}-\mathrm{C} 9 \mathrm{~B}-\mathrm{H} 9 \mathrm{~B}$ & 119.2 \\
\hline $\mathrm{C} 8-\mathrm{C} 9-\mathrm{H} 9$ & 119.3 & $\mathrm{C} 4 \mathrm{~B}-\mathrm{C} 9 \mathrm{~B}-\mathrm{H} 9 \mathrm{~B}$ & 119.2 \\
\hline $\mathrm{C} 4-\mathrm{C} 9-\mathrm{H} 9$ & 119.3 & $\mathrm{C} 15 \mathrm{~B}-\mathrm{C} 10 \mathrm{~B}-\mathrm{C} 11 \mathrm{~B}$ & $120.5(6)$ \\
\hline $\mathrm{C} 15-\mathrm{C} 10-\mathrm{C} 11$ & $119.1(5)$ & $\mathrm{C} 15 \mathrm{~B}-\mathrm{C} 10 \mathrm{~B}-\mathrm{P} 1 \mathrm{~B}$ & $119.9(5)$ \\
\hline $\mathrm{C} 15-\mathrm{C} 10-\mathrm{P} 1$ & $121.6(4)$ & $\mathrm{C} 11 \mathrm{~B}-\mathrm{C} 10 \mathrm{~B}-\mathrm{P} 1 \mathrm{~B}$ & $119.5(5)$ \\
\hline $\mathrm{C} 11-\mathrm{C} 10-\mathrm{P} 1$ & $119.3(4)$ & $\mathrm{C} 12 \mathrm{~B}-\mathrm{C} 11 \mathrm{~B}-\mathrm{C} 10 \mathrm{~B}$ & $120.1(7)$ \\
\hline
\end{tabular}




\begin{tabular}{|c|c|c|c|}
\hline $\mathrm{C} 10-\mathrm{C} 11-\mathrm{C} 12$ & $119.5(5)$ & $\mathrm{C} 12 \mathrm{~B}-\mathrm{C} 11 \mathrm{~B}-\mathrm{H} 11 \mathrm{~B}$ & 120 \\
\hline $\mathrm{C} 10-\mathrm{C} 11-\mathrm{H} 11$ & 120.2 & $\mathrm{C} 10 \mathrm{~B}-\mathrm{C} 11 \mathrm{~B}-\mathrm{H} 11 \mathrm{~B}$ & 120 \\
\hline $\mathrm{C} 12-\mathrm{C} 11-\mathrm{H} 11$ & 120.2 & $\mathrm{C} 13 \mathrm{~B}-\mathrm{C} 12 \mathrm{~B}-\mathrm{C} 11 \mathrm{~B}$ & $119.2(7)$ \\
\hline $\mathrm{C} 13-\mathrm{C} 12-\mathrm{C} 11$ & $121.0(5)$ & $\mathrm{C} 13 \mathrm{~B}-\mathrm{C} 12 \mathrm{~B}-\mathrm{H} 12 \mathrm{~B}$ & 120.4 \\
\hline $\mathrm{C} 13-\mathrm{C} 12-\mathrm{H} 12$ & 119.5 & $\mathrm{C} 11 \mathrm{~B}-\mathrm{C} 12 \mathrm{~B}-\mathrm{H} 12 \mathrm{~B}$ & 120.4 \\
\hline $\mathrm{C} 11-\mathrm{C} 12-\mathrm{H} 12$ & 119.5 & $\mathrm{C} 12 \mathrm{~B}-\mathrm{C} 13 \mathrm{~B}-\mathrm{C} 14 \mathrm{~B}$ & $122.5(7)$ \\
\hline $\mathrm{C} 14-\mathrm{C} 13-\mathrm{C} 12$ & $119.6(5)$ & $\mathrm{C} 12 \mathrm{~B}-\mathrm{C} 13 \mathrm{~B}-\mathrm{H} 13 \mathrm{~B}$ & 118.7 \\
\hline $\mathrm{C} 14-\mathrm{C} 13-\mathrm{H} 13$ & 120.2 & $\mathrm{C} 14 \mathrm{~B}-\mathrm{C} 13 \mathrm{~B}-\mathrm{H} 13 \mathrm{~B}$ & 118.7 \\
\hline $\mathrm{C} 12-\mathrm{C} 13-\mathrm{H} 13$ & 120.2 & $\mathrm{C} 13 \mathrm{~B}-\mathrm{C} 14 \mathrm{~B}-\mathrm{C} 15 \mathrm{~B}$ & $119.6(8)$ \\
\hline $\mathrm{C} 13-\mathrm{C} 14-\mathrm{C} 15$ & $120.4(6)$ & $\mathrm{C} 13 \mathrm{~B}-\mathrm{C} 14 \mathrm{~B}-\mathrm{H} 14 \mathrm{~B}$ & 120.2 \\
\hline $\mathrm{C} 13-\mathrm{C} 14-\mathrm{H} 14$ & 119.8 & $\mathrm{C} 15 \mathrm{~B}-\mathrm{C} 14 \mathrm{~B}-\mathrm{H} 14 \mathrm{~B}$ & 120.2 \\
\hline $\mathrm{C} 15-\mathrm{C} 14-\mathrm{H} 14$ & 119.8 & $\mathrm{C} 10 \mathrm{~B}-\mathrm{C} 15 \mathrm{~B}-\mathrm{C} 14 \mathrm{~B}$ & $118.0(7)$ \\
\hline $\mathrm{C} 10-\mathrm{C} 15-\mathrm{C} 14$ & $120.3(5)$ & $\mathrm{C} 10 \mathrm{~B}-\mathrm{C} 15 \mathrm{~B}-\mathrm{H} 15 \mathrm{~B}$ & 121 \\
\hline $\mathrm{C} 10-\mathrm{C} 15-\mathrm{H} 15$ & 119.9 & $\mathrm{C} 14 \mathrm{~B}-\mathrm{C} 15 \mathrm{~B}-\mathrm{H} 15 \mathrm{~B}$ & 121 \\
\hline $\mathrm{C} 14-\mathrm{C} 15-\mathrm{H} 15$ & 119.9 & $\mathrm{C} 21 \mathrm{~B}-\mathrm{C} 16 \mathrm{~B}-\mathrm{C} 17 \mathrm{~B}$ & $118.3(5)$ \\
\hline $\mathrm{C} 17-\mathrm{C} 16-\mathrm{C} 21$ & $119.1(5)$ & $\mathrm{C} 21 \mathrm{~B}-\mathrm{C} 16 \mathrm{~B}-\mathrm{P} 2 \mathrm{~B}$ & $122.9(4)$ \\
\hline $\mathrm{C} 17-\mathrm{C} 16-\mathrm{P} 2$ & $118.8(4)$ & $\mathrm{C} 17 \mathrm{~B}-\mathrm{C} 16 \mathrm{~B}-\mathrm{P} 2 \mathrm{~B}$ & $118.7(4)$ \\
\hline $\mathrm{C} 21-\mathrm{C} 16-\mathrm{P} 2$ & $121.9(4)$ & $\mathrm{C} 18 \mathrm{~B}-\mathrm{C} 17 \mathrm{~B}-\mathrm{C} 16 \mathrm{~B}$ & $120.7(5)$ \\
\hline $\mathrm{C} 16-\mathrm{C} 17-\mathrm{C} 18$ & $121.3(5)$ & $\mathrm{C} 18 \mathrm{~B}-\mathrm{C} 17 \mathrm{~B}-\mathrm{H} 17 \mathrm{~B}$ & 119.7 \\
\hline $\mathrm{C} 16-\mathrm{C} 17-\mathrm{H} 17$ & 119.4 & $\mathrm{C} 16 \mathrm{~B}-\mathrm{C} 17 \mathrm{~B}-\mathrm{H} 17 \mathrm{~B}$ & 119.7 \\
\hline $\mathrm{C} 18-\mathrm{C} 17-\mathrm{H} 17$ & 119.4 & $\mathrm{C} 17 \mathrm{~B}-\mathrm{C} 18 \mathrm{~B}-\mathrm{C} 19 \mathrm{~B}$ & $121.9(6)$ \\
\hline $\mathrm{C} 19-\mathrm{C} 18-\mathrm{C} 17$ & $119.0(5)$ & $\mathrm{C} 17 \mathrm{~B}-\mathrm{C} 18 \mathrm{~B}-\mathrm{H} 18 \mathrm{~B}$ & 119 \\
\hline $\mathrm{C} 19-\mathrm{C} 18-\mathrm{H} 18$ & 120.5 & $\mathrm{C} 19 \mathrm{~B}-\mathrm{C} 18 \mathrm{~B}-\mathrm{H} 18 \mathrm{~B}$ & 119 \\
\hline $\mathrm{C} 17-\mathrm{C} 18-\mathrm{H} 18$ & 120.5 & $\mathrm{C} 18 \mathrm{~B}-\mathrm{C} 19 \mathrm{~B}-\mathrm{C} 20 \mathrm{~B}$ & $119.1(5)$ \\
\hline $\mathrm{C} 18-\mathrm{C} 19-\mathrm{C} 20$ & $120.9(5)$ & $\mathrm{C} 18 \mathrm{~B}-\mathrm{C} 19 \mathrm{~B}-\mathrm{H} 19 \mathrm{~B}$ & 120.5 \\
\hline $\mathrm{C} 18-\mathrm{C} 19-\mathrm{H} 19$ & 119.6 & $\mathrm{C} 20 \mathrm{~B}-\mathrm{C} 19 \mathrm{~B}-\mathrm{H} 19 \mathrm{~B}$ & 120.5 \\
\hline $\mathrm{C} 20-\mathrm{C} 19-\mathrm{H} 19$ & 119.6 & $\mathrm{C} 21 \mathrm{~B}-\mathrm{C} 20 \mathrm{~B}-\mathrm{C} 19 \mathrm{~B}$ & $120.4(5)$ \\
\hline $\mathrm{C} 19-\mathrm{C} 20-\mathrm{C} 21$ & $119.4(5)$ & $\mathrm{C} 21 \mathrm{~B}-\mathrm{C} 20 \mathrm{~B}-\mathrm{H} 20 \mathrm{~B}$ & 119.8 \\
\hline $\mathrm{C} 19-\mathrm{C} 20-\mathrm{H} 20$ & 120.3 & $\mathrm{C} 19 \mathrm{~B}-\mathrm{C} 20 \mathrm{~B}-\mathrm{H} 20 \mathrm{~B}$ & 119.8 \\
\hline $\mathrm{C} 21-\mathrm{C} 20-\mathrm{H} 20$ & 120.3 & $\mathrm{C} 20 \mathrm{~B}-\mathrm{C} 21 \mathrm{~B}-\mathrm{C} 16 \mathrm{~B}$ & $119.6(5)$ \\
\hline $\mathrm{C} 16-\mathrm{C} 21-\mathrm{C} 20$ & $120.3(5)$ & $\mathrm{C} 20 \mathrm{~B}-\mathrm{C} 21 \mathrm{~B}-\mathrm{H} 21 \mathrm{~B}$ & 120.2 \\
\hline $\mathrm{C} 16-\mathrm{C} 21-\mathrm{H} 21$ & 119.9 & $\mathrm{C} 16 \mathrm{~B}-\mathrm{C} 21 \mathrm{~B}-\mathrm{H} 21 \mathrm{~B}$ & 120.2 \\
\hline $\mathrm{C} 20-\mathrm{C} 21-\mathrm{H} 21$ & 119.9 & $\mathrm{C} 23 \mathrm{~B}-\mathrm{C} 22 \mathrm{~B}-\mathrm{C} 27 \mathrm{~B}$ & $118.4(5)$ \\
\hline $\mathrm{C} 23-\mathrm{C} 22-\mathrm{C} 27$ & $119.2(5)$ & $\mathrm{C} 23 \mathrm{~B}-\mathrm{C} 22 \mathrm{~B}-\mathrm{P} 2 \mathrm{~B}$ & $122.2(4)$ \\
\hline $\mathrm{C} 23-\mathrm{C} 22-\mathrm{P} 2$ & $122.0(4)$ & $\mathrm{C} 27 \mathrm{~B}-\mathrm{C} 22 \mathrm{~B}-\mathrm{P} 2 \mathrm{~B}$ & $119.3(4)$ \\
\hline $\mathrm{C} 27-\mathrm{C} 22-\mathrm{P} 2$ & $118.8(4)$ & $\mathrm{C} 22 \mathrm{~B}-\mathrm{C} 23 \mathrm{~B}-\mathrm{C} 24 \mathrm{~B}$ & $119.9(6)$ \\
\hline $\mathrm{C} 22-\mathrm{C} 23-\mathrm{C} 24$ & $120.7(5)$ & $\mathrm{C} 22 \mathrm{~B}-\mathrm{C} 23 \mathrm{~B}-\mathrm{H} 23 \mathrm{~B}$ & 120.1 \\
\hline $\mathrm{C} 22-\mathrm{C} 23-\mathrm{H} 23$ & 119.7 & $\mathrm{C} 24 \mathrm{~B}-\mathrm{C} 23 \mathrm{~B}-\mathrm{H} 23 \mathrm{~B}$ & 120.1 \\
\hline $\mathrm{C} 24-\mathrm{C} 23-\mathrm{H} 23$ & 119.7 & $\mathrm{C} 25 \mathrm{~B}-\mathrm{C} 24 \mathrm{~B}-\mathrm{C} 23 \mathrm{~B}$ & $121.7(6)$ \\
\hline $\mathrm{C} 25-\mathrm{C} 24-\mathrm{C} 23$ & $119.5(6)$ & $\mathrm{C} 25 \mathrm{~B}-\mathrm{C} 24 \mathrm{~B}-\mathrm{H} 24 \mathrm{~B}$ & 119.1 \\
\hline $\mathrm{C} 25-\mathrm{C} 24-\mathrm{H} 24$ & 120.2 & $\mathrm{C} 23 \mathrm{~B}-\mathrm{C} 24 \mathrm{~B}-\mathrm{H} 24 \mathrm{~B}$ & 119.1 \\
\hline $\mathrm{C} 23-\mathrm{C} 24-\mathrm{H} 24$ & 120.2 & $\mathrm{C} 24 \mathrm{~B}-\mathrm{C} 25 \mathrm{~B}-\mathrm{C} 26 \mathrm{~B}$ & $118.9(6)$ \\
\hline $\mathrm{C} 26-\mathrm{C} 25-\mathrm{C} 24$ & $120.6(6)$ & $\mathrm{C} 24 \mathrm{~B}-\mathrm{C} 25 \mathrm{~B}-\mathrm{H} 25 \mathrm{~B}$ & 120.6 \\
\hline $\mathrm{C} 26-\mathrm{C} 25-\mathrm{H} 25$ & 119.7 & $\mathrm{C} 26 \mathrm{~B}-\mathrm{C} 25 \mathrm{~B}-\mathrm{H} 25 \mathrm{~B}$ & 120.6 \\
\hline $\mathrm{C} 24-\mathrm{C} 25-\mathrm{H} 25$ & 119.7 & $\mathrm{C} 27 \mathrm{~B}-\mathrm{C} 26 \mathrm{~B}-\mathrm{C} 25 \mathrm{~B}$ & $120.4(6)$ \\
\hline $\mathrm{C} 25-\mathrm{C} 26-\mathrm{C} 27$ & $120.0(6)$ & $\mathrm{C} 27 \mathrm{~B}-\mathrm{C} 26 \mathrm{~B}-\mathrm{H} 26 \mathrm{~B}$ & 119.8 \\
\hline $\mathrm{C} 25-\mathrm{C} 26-\mathrm{H} 26$ & 120 & $\mathrm{C} 25 \mathrm{~B}-\mathrm{C} 26 \mathrm{~B}-\mathrm{H} 26 \mathrm{~B}$ & 119.8 \\
\hline $\mathrm{C} 27-\mathrm{C} 26-\mathrm{H} 26$ & 120 & $\mathrm{C} 26 \mathrm{~B}-\mathrm{C} 27 \mathrm{~B}-\mathrm{C} 22 \mathrm{~B}$ & $120.6(6)$ \\
\hline
\end{tabular}




\begin{tabular}{|c|c|}
\hline $\mathrm{C} 22-\mathrm{C} 27-\mathrm{C} 26$ & $120.0(5)$ \\
\hline $\mathrm{C} 22-\mathrm{C} 27-\mathrm{H} 27$ & 120 \\
\hline $\mathrm{C} 26-\mathrm{C} 27-\mathrm{H} 27$ & 120 \\
\hline $\mathrm{C} 29-\mathrm{C} 28-\mathrm{N}$ & $111.9(4)$ \\
\hline $\mathrm{C} 29-\mathrm{C} 28-\mathrm{H} 28 \mathrm{~A}$ & 109.2 \\
\hline $\mathrm{N}-\mathrm{C} 28-\mathrm{H} 28 \mathrm{~A}$ & 109.2 \\
\hline $\mathrm{C} 29-\mathrm{C} 28-\mathrm{H} 28 \mathrm{~B}$ & 109.2 \\
\hline $\mathrm{N}-\mathrm{C} 28-\mathrm{H} 28 \mathrm{~B}$ & 109.2 \\
\hline $\mathrm{H} 28 \mathrm{~A}-\mathrm{C} 28-\mathrm{H} 28 \mathrm{~B}$ & 107.9 \\
\hline $\mathrm{C} 28-\mathrm{C} 29-\mathrm{S} 1$ & $111.9(4)$ \\
\hline $\mathrm{C} 28-\mathrm{C} 29-\mathrm{H} 29 \mathrm{~A}$ & 109.2 \\
\hline $\mathrm{S} 1-\mathrm{C} 29-\mathrm{H} 29 \mathrm{~A}$ & 109.2 \\
\hline $\mathrm{C} 28-\mathrm{C} 29-\mathrm{H} 29 \mathrm{~B}$ & 109.2 \\
\hline $\mathrm{S} 1-\mathrm{C} 29-\mathrm{H} 29 \mathrm{~B}$ & 109.2 \\
\hline $\mathrm{H} 29 \mathrm{~A}-\mathrm{C} 29-\mathrm{H} 29 \mathrm{~B}$ & 107.9 \\
\hline $\mathrm{N}-\mathrm{C} 30-\mathrm{C} 31$ & $109.8(4)$ \\
\hline $\mathrm{N}-\mathrm{C} 30-\mathrm{H} 30 \mathrm{~A}$ & 109.7 \\
\hline $\mathrm{C} 31-\mathrm{C} 30-\mathrm{H} 30 \mathrm{~A}$ & 109.7 \\
\hline $\mathrm{N}-\mathrm{C} 30-\mathrm{H} 30 \mathrm{~B}$ & 109.7 \\
\hline $\mathrm{C} 31-\mathrm{C} 30-\mathrm{H} 30 \mathrm{~B}$ & 109.7 \\
\hline $\mathrm{H} 30 \mathrm{~A}-\mathrm{C} 30-\mathrm{H} 30 \mathrm{~B}$ & 108.2 \\
\hline $\mathrm{C} 30-\mathrm{C} 31-\mathrm{S} 2$ & $109.7(4)$ \\
\hline $\mathrm{C} 30-\mathrm{C} 31-\mathrm{H} 31 \mathrm{~A}$ & 109.7 \\
\hline $\mathrm{S} 2-\mathrm{C} 31-\mathrm{H} 31 \mathrm{~A}$ & 109.7 \\
\hline $\mathrm{C} 30-\mathrm{C} 31-\mathrm{H} 31 \mathrm{~B}$ & 109.7 \\
\hline $\mathrm{S} 2-\mathrm{C} 31-\mathrm{H} 31 \mathrm{~B}$ & 109.7 \\
\hline $\mathrm{H} 31 \mathrm{~A}-\mathrm{C} 31-\mathrm{H} 31 \mathrm{~B}$ & 108.2 \\
\hline $\mathrm{N}-\mathrm{C} 32-\mathrm{C} 33$ & $114.1(5)$ \\
\hline $\mathrm{N}-\mathrm{C} 32-\mathrm{H} 32 \mathrm{~A}$ & 108.7 \\
\hline $\mathrm{C} 33-\mathrm{C} 32-\mathrm{H} 32 \mathrm{~A}$ & 108.7 \\
\hline $\mathrm{N}-\mathrm{C} 32-\mathrm{H} 32 \mathrm{~B}$ & 108.7 \\
\hline C33-C32-H32B & 108.7 \\
\hline $\mathrm{H} 32 \mathrm{~A}-\mathrm{C} 32-\mathrm{H} 32 \mathrm{~B}$ & 107.6 \\
\hline $\mathrm{C} 32-\mathrm{C} 33-\mathrm{S} 3$ & $110.6(4)$ \\
\hline $\mathrm{C} 32-\mathrm{C} 33-\mathrm{H} 33 \mathrm{~A}$ & 109.5 \\
\hline $\mathrm{S} 3-\mathrm{C} 33-\mathrm{H} 33 \mathrm{~A}$ & 109.5 \\
\hline $\mathrm{C} 32-\mathrm{C} 33-\mathrm{H} 33 \mathrm{~B}$ & 109.5 \\
\hline $\mathrm{S} 3-\mathrm{C} 33-\mathrm{H} 33 \mathrm{~B}$ & 109.5 \\
\hline $\mathrm{H} 33 \mathrm{~A}-\mathrm{C} 33-\mathrm{H} 33 \mathrm{~B}$ & 108.1 \\
\hline $\mathrm{O} 1-\mathrm{C} 34-\mathrm{Fe}$ & $175.3(5)$ \\
\hline $\mathrm{O} 2-\mathrm{C} 35-\mathrm{Fe}$ & $176.9(5)$ \\
\hline $\mathrm{P} 1 \mathrm{~B}-\mathrm{Ni1B}-\mathrm{P} 2 \mathrm{~B}$ & $92.84(6)$ \\
\hline $\mathrm{Fe}-\mathrm{S} 1-\mathrm{C} 29-\mathrm{C} 28$ & $-9.4(6)$ \\
\hline $\mathrm{S} 1-\mathrm{C} 29-\mathrm{C} 28-\mathrm{N}$ & $38.6(8)$ \\
\hline $\mathrm{C} 29-\mathrm{C} 28-\mathrm{N}-\mathrm{Fe}$ & $-51.9(7)$ \\
\hline $\mathrm{Fe}-\mathrm{S} 2-\mathrm{C} 31-\mathrm{C} 30$ & $23.7(6)$ \\
\hline $\mathrm{S} 2-\mathrm{C} 31-\mathrm{C} 30-\mathrm{N}$ & $-51.7(8)$ \\
\hline
\end{tabular}

\begin{tabular}{|c|c|}
\hline $\mathrm{C} 26 \mathrm{~B}-\mathrm{C} 27 \mathrm{~B}-\mathrm{H} 27 \mathrm{~B}$ & 119.7 \\
\hline $\mathrm{C} 22 \mathrm{~B}-\mathrm{C} 27 \mathrm{~B}-\mathrm{H} 27 \mathrm{~B}$ & 119.7 \\
\hline $\mathrm{C} 29 \mathrm{~B}-\mathrm{C} 28 \mathrm{~B}-\mathrm{N} 1 \mathrm{~B}$ & $117.8(6)$ \\
\hline $\mathrm{C} 29 \mathrm{~B}-\mathrm{C} 28 \mathrm{~B}-\mathrm{H} 28 \mathrm{C}$ & 107.9 \\
\hline $\mathrm{N} 1 \mathrm{~B}-\mathrm{C} 28 \mathrm{~B}-\mathrm{H} 28 \mathrm{C}$ & 107.9 \\
\hline $\mathrm{C} 29 \mathrm{~B}-\mathrm{C} 28 \mathrm{~B}-\mathrm{H} 28 \mathrm{D}$ & 107.9 \\
\hline N1B-C28B-H28D & 107.9 \\
\hline $\mathrm{H} 28 \mathrm{C}-\mathrm{C} 28 \mathrm{~B}-\mathrm{H} 28 \mathrm{D}$ & 107.2 \\
\hline $\mathrm{C} 28 \mathrm{~B}-\mathrm{C} 29 \mathrm{~B}-\mathrm{S} 1 \mathrm{~B}$ & $113.7(5)$ \\
\hline $\mathrm{C} 28 \mathrm{~B}-\mathrm{C} 29 \mathrm{~B}-\mathrm{H} 29 \mathrm{C}$ & 108.8 \\
\hline $\mathrm{S} 1 \mathrm{~B}-\mathrm{C} 29 \mathrm{~B}-\mathrm{H} 29 \mathrm{C}$ & 108.8 \\
\hline $\mathrm{C} 28 \mathrm{~B}-\mathrm{C} 29 \mathrm{~B}-\mathrm{H} 29 \mathrm{D}$ & 108.8 \\
\hline $\mathrm{S} 1 \mathrm{~B}-\mathrm{C} 29 \mathrm{~B}-\mathrm{H} 29 \mathrm{D}$ & 108.8 \\
\hline $\mathrm{H} 29 \mathrm{C}-\mathrm{C} 29 \mathrm{~B}-\mathrm{H} 29 \mathrm{D}$ & 107.7 \\
\hline $\mathrm{N} 1 \mathrm{~B}-\mathrm{C} 30 \mathrm{~B}-\mathrm{C} 31 \mathrm{~B}$ & $106.4(5)$ \\
\hline $\mathrm{N} 1 \mathrm{~B}-\mathrm{C} 30 \mathrm{~B}-\mathrm{H} 30 \mathrm{C}$ & 110.5 \\
\hline $\mathrm{C} 31 \mathrm{~B}-\mathrm{C} 30 \mathrm{~B}-\mathrm{H} 30 \mathrm{C}$ & 110.5 \\
\hline $\mathrm{N} 1 \mathrm{~B}-\mathrm{C} 30 \mathrm{~B}-\mathrm{H} 30 \mathrm{D}$ & 110.5 \\
\hline $\mathrm{C} 31 \mathrm{~B}-\mathrm{C} 30 \mathrm{~B}-\mathrm{H} 30 \mathrm{D}$ & 110.5 \\
\hline $\mathrm{H} 30 \mathrm{C}-\mathrm{C} 30 \mathrm{~B}-\mathrm{H} 30 \mathrm{D}$ & 108.6 \\
\hline $\mathrm{C} 30 \mathrm{~B}-\mathrm{C} 31 \mathrm{~B}-\mathrm{S} 2 \mathrm{~B}$ & $108.4(5)$ \\
\hline $\mathrm{C} 30 \mathrm{~B}-\mathrm{C} 31 \mathrm{~B}-\mathrm{H} 31 \mathrm{C}$ & 110 \\
\hline $\mathrm{S} 2 \mathrm{~B}-\mathrm{C} 31 \mathrm{~B}-\mathrm{H} 31 \mathrm{C}$ & 110 \\
\hline $\mathrm{C} 30 \mathrm{~B}-\mathrm{C} 31 \mathrm{~B}-\mathrm{H} 31 \mathrm{D}$ & 110 \\
\hline $\mathrm{S} 2 \mathrm{~B}-\mathrm{C} 31 \mathrm{~B}-\mathrm{H} 31 \mathrm{D}$ & 110 \\
\hline $\mathrm{H} 31 \mathrm{C}-\mathrm{C} 31 \mathrm{~B}-\mathrm{H} 31 \mathrm{D}$ & 108.4 \\
\hline $\mathrm{C} 33 \mathrm{~B}-\mathrm{C} 32 \mathrm{~B}-\mathrm{N} 1 \mathrm{~B}$ & $115.4(5)$ \\
\hline $\mathrm{C} 33 \mathrm{~B}-\mathrm{C} 32 \mathrm{~B}-\mathrm{H} 32 \mathrm{C}$ & 108.4 \\
\hline $\mathrm{N} 1 \mathrm{~B}-\mathrm{C} 32 \mathrm{~B}-\mathrm{H} 32 \mathrm{C}$ & 108.4 \\
\hline $\mathrm{C} 33 \mathrm{~B}-\mathrm{C} 32 \mathrm{~B}-\mathrm{H} 32 \mathrm{D}$ & 108.4 \\
\hline $\mathrm{N} 1 \mathrm{~B}-\mathrm{C} 32 \mathrm{~B}-\mathrm{H} 32 \mathrm{D}$ & 108.4 \\
\hline $\mathrm{H} 32 \mathrm{C}-\mathrm{C} 32 \mathrm{~B}-\mathrm{H} 32 \mathrm{D}$ & 107.5 \\
\hline $\mathrm{C} 32 \mathrm{~B}-\mathrm{C} 33 \mathrm{~B}-\mathrm{S} 3 \mathrm{~B}$ & $112.3(4)$ \\
\hline $\mathrm{C} 32 \mathrm{~B}-\mathrm{C} 33 \mathrm{~B}-\mathrm{H} 33 \mathrm{C}$ & 109.1 \\
\hline $\mathrm{S} 3 \mathrm{~B}-\mathrm{C} 33 \mathrm{~B}-\mathrm{H} 33 \mathrm{C}$ & 109.1 \\
\hline $\mathrm{C} 32 \mathrm{~B}-\mathrm{C} 33 \mathrm{~B}-\mathrm{H} 33 \mathrm{D}$ & 109.1 \\
\hline $\mathrm{S} 3 \mathrm{~B}-\mathrm{C} 33 \mathrm{~B}-\mathrm{H} 33 \mathrm{D}$ & 109.1 \\
\hline $\mathrm{H} 33 \mathrm{C}-\mathrm{C} 33 \mathrm{~B}-\mathrm{H} 33 \mathrm{D}$ & 107.9 \\
\hline $\mathrm{O} 1 \mathrm{~B}-\mathrm{C} 34 \mathrm{~B}-\mathrm{Fe} 2 \mathrm{~B}$ & $175.4(8)$ \\
\hline $\mathrm{O} 2 \mathrm{~B}-\mathrm{C} 35 \mathrm{~B}-\mathrm{Fe} 2 \mathrm{~B}$ & $176.9(5)$ \\
\hline $\mathrm{N} 2-\mathrm{C} 37-\mathrm{C} 36$ & $175.4(9)$ \\
\hline $\mathrm{N} 2 \mathrm{~B}-\mathrm{C} 37 \mathrm{~B}-\mathrm{C} 36 \mathrm{~B}$ & $178.0(10)$ \\
\hline $\mathrm{C} 29 \mathrm{~B}-\mathrm{C} 28 \mathrm{~B}-\mathrm{N} 1 \mathrm{~B}-\mathrm{Fe} 2 \mathrm{~B}$ & $-43.0(10$ \\
\hline $\mathrm{Fe} 2 \mathrm{~B}-\mathrm{S} 2 \mathrm{~B}-\mathrm{C} 31 \mathrm{~B}-\mathrm{C} 30 \mathrm{~B}$ & $25.8(8)$ \\
\hline $\mathrm{S} 2 \mathrm{~B}-\mathrm{C} 31 \mathrm{~B}-\mathrm{C} 30 \mathrm{~B}-\mathrm{N} 1 \mathrm{~B}$ & $-54.9(10$ \\
\hline $\mathrm{C} 31 \mathrm{~B}-\mathrm{C} 30 \mathrm{~B}-\mathrm{N} 1 \mathrm{~B}-\mathrm{Fe} 2 \mathrm{~B}$ & $58.7(9)$ \\
\hline $\mathrm{Fe} 2 \mathrm{~B}-\mathrm{S} 3 \mathrm{~B}-\mathrm{C} 33 \mathrm{~B}-\mathrm{C} 32 \mathrm{~B}$ & $-35.8(7)$ \\
\hline
\end{tabular}




$\begin{array}{llll}\mathrm{C} 31-\mathrm{C} 30-\mathrm{N}-\mathrm{Fe} & 55.3(7) & \mathrm{S} 3 \mathrm{~B}-\mathrm{C} 33 \mathrm{~B}-\mathrm{C} 32 \mathrm{~B}-\mathrm{N} 1 \mathrm{~B} & 42.1(10) \\ \mathrm{Fe}-\mathrm{S} 3-\mathrm{C} 33-\mathrm{C} 32 & -38.3(6) & \mathrm{C} 33 \mathrm{~B}-\mathrm{C} 32 \mathrm{~B}-\mathrm{N} 1 \mathrm{~B}-\mathrm{Fe} 2 \mathrm{~B} & -24.5(10) \\ \mathrm{S} 3-\mathrm{C} 33-\mathrm{C} 32-\mathrm{N} & 45.8(8) & \mathrm{P} 1-\mathrm{C} 1-\mathrm{C} 2-\mathrm{C} 3 & -68.5(8) \\ \mathrm{C} 33-\mathrm{C} 32-\mathrm{N}-\mathrm{Fe} & -27.3(8) & \mathrm{C} 1-\mathrm{C} 2-\mathrm{C} 3-\mathrm{P} 2 & 69.7(8) \\ \mathrm{Fe} 2 \mathrm{~B}-\mathrm{S} 1 \mathrm{~B}-\mathrm{C} 29 \mathrm{~B}-\mathrm{C} 28 \mathrm{~B} & -3.0(8) & \mathrm{P} 1 \mathrm{~B}-\mathrm{C} 1 \mathrm{~B}-\mathrm{C} 2 \mathrm{~B}-\mathrm{C} 3 \mathrm{~B} & -69.3(8) \\ \mathrm{S} 1 \mathrm{~B}-\mathrm{C} 29 \mathrm{~B}-\mathrm{C} 28 \mathrm{~B}-\mathrm{N} 1 \mathrm{~B} & 29.6(12) & \mathrm{C} 1 \mathrm{~B}-\mathrm{C} 2 \mathrm{~B}-\mathrm{C} 3 \mathrm{~B}-\mathrm{P} 2 \mathrm{~B} & 67.1(8)\end{array}$

